\title{
MORE LECTURES ON HILBERT SCHEMES OF POINTS ON SURFACES
}

\author{
HIRAKU NAKAJIMA \\ Dedicated to Professor Shigeru Mukai on the occasion of his 60th birthday
}

\section{INTRODUCTION}

This paper is based on author's lectures at Kyoto University in 2010 Summer, and in the 6th MSJ-SI 'Development of Moduli Theory' at RIMS in June 2013.

The purpose of lectures was to review several results on Hilbert schemes of points which were obtained after author's lecture note [24] was written. Among many results, we choose those which are about equivariant homology groups $H_{*}^{T}\left(X^{[n]}\right)$ of Hilbert schemes of points on the affine plane $X=\mathbb{C}^{2}$ with respect to the torus action. Study of equivariant homology groups increases its importance recently. In particular, it is a basis of the AGT correspondence between instanton moduli spaces on $\mathbb{C}^{2}$ and the representation theory of $W$-algebras, which is a very hot topic now (see e.g., [20]).

We omit proofs if they are present in [24], but give self-contained proofs otherwise. In this sense, this should be read after [24].

The paper is organized as follows. In $\S 1$, we review basics on equivariant (co)homology groups. It will be basis of subsequent sections. In $\S 2$ we construct the Fock representation of the Heisenberg algebra on $\bigoplus H_{*}^{T}\left(X^{[n]}\right)$, following [24, Ch. 8] as well as an idea of Vasserot [27]. In $\S 3$ we explain a geometric realization of Jack symmetric functions as fixed point classes in $H_{*}^{T}\left(X^{[n]}\right)$ by Li-Qin-Wang [17]. We also give author's unpublished result, which was used in [17]. As applications, we give geometric proofs of the norm formula and Pieri formula of Jack symmetric functions. In $\S 4$ we construct a representation of the Virasoro algebra on $\bigoplus H_{*}^{T}\left(X^{[n]}\right)$. It is a special case of Lehn's result [14] for $X=\mathbb{C}^{2}$, but the proof is different.

2000 Mathematics Subject Classification. Primary 14C05; Secondary 14D21, 14J60. 
Acknowledgment. This work was supported by the Grant-in-Aid for Scientific Research (B) (No. 23340005), Japan Society for the Promotion of Science.

\section{Equivariant (CO)homology Groups}

In this section, we review basics on equivariant cohomology and homology groups, which will be used in later sections. Our definition of equivariant cohomology groups is slightly different from the usual one (e.g., as in [1]). We replace the classifying space BT by its finite dimensional approximations. This approach is suitable for equivariant homology groups, and was taken by Lusztig [18]. The same approach was used for equivariant Chow groups [8].

We assume coefficients of cohomology groups are $\mathbb{C}$.

1(i). Equivariant cohomology groups. Let $T=\left(\mathbb{C}^{*}\right)^{r}$ be an algebraic torus. Let $V=\left(\mathbb{C}^{N+1}\right)^{r}$ be a $T$-module, where each $\mathbb{C}^{*}$ acts on the corresponding $\mathbb{C}^{N}$ by multiplication. Then $V \backslash\{0\} \rightarrow\left(\mathbb{P}^{N}\right)^{r}$ is a $T$-bundle, and the universal $T$-bundle $E T \rightarrow B T$ is the inductive limit when $N \rightarrow \infty$. Let $M$ be a $T$-variety, i.e., an algebraic variety with an algebraic $T$-action. We further assume that $M$ admits a locally closed $T$-embedding into a smooth projective $T$-variety. This restriction can be weakened, but it is enough for our purpose.

We define the equivariant cohomology of $M$ by

$$
H_{T}^{i}(M) \stackrel{\text { def. }}{=} H^{i}\left(M_{V}\right) \quad \text { where } M_{V}=(V \backslash\{0\}) \times_{T} M .
$$

Here for a given $i$, we take $V$ with sufficiently large $N$. Then it is well-defined thanks to the following lemma:

Lemma 1.1. $H_{T}^{i}(M)$ is independent of the choice of $V$.

Proof. A key point is that $H^{i}(V \backslash\{0\}) \cong H^{i}\left(\left(S^{2 N+1}\right)^{r}\right)=0$ if $0<i<$ $2 N+1$.

Take two large vector spaces $V_{1}, V_{2}$ and consider the diagram

$$
M_{V_{1}} \leftarrow\left(V_{1} \backslash\{0\} \times V_{2} \backslash\{0\}\right) \times_{T} M \rightarrow M_{V_{2}} .
$$

The left and right arrows are fiber bundles with fibers $V_{2} \backslash\{0\}, V_{1} \backslash\{0\}$ respectively. Since their cohomology groups vanishes in degree between 1 and sufficiently large number (in particular, larger than $i$ ), $H^{i}\left(M_{V_{1}}\right)$ and $H^{i}\left(M_{V_{2}}\right)$ are isomorphic to $H^{i}$ of the middle under the pull-back homomorphisms.

Let us briefly explain several important properties of equivariant cohomology groups. 
When $M=$ pt, we have $H_{T}^{i}(\mathrm{pt})=H^{i}\left(\left(\mathbb{P}^{N}\right)^{r}\right)$. Note that $H^{*}\left(\mathbb{P}^{N}\right)=$ $\mathbb{C}[a] /\left(a^{N+1}=0\right)$. Taking $N \rightarrow \infty$, we have

$$
H_{T}^{*}(\mathrm{pt}) \cong \mathbb{C}\left[a_{1}, \ldots, a_{r}\right]
$$

where $a_{i}$ is the first Chern class of the hyperplane bundle $\mathcal{O}(1)$ of the $i^{\text {th }}$ factor of $\left(\mathbb{P}^{N}\right)^{r}$. As this example shows, $H_{T}^{i}(M)$ may be nonzero in arbitrary large degree $i$ unlike ordinary cohomology groups.

When $T$ acts trivially on $M$, we have

$$
H_{T}^{*}(M) \cong H^{*}(M) \otimes H_{T}^{*}(\mathrm{pt})
$$

from the definition.

We have a cup product

$$
H_{T}^{i}(M) \otimes H_{T}^{j}(M) \rightarrow H_{T}^{i+j}(M) .
$$

The isomorphism (1.2) is a ring isomorphism.

If $f: M_{1} \rightarrow M_{2}$ is a $T$-equivariant continuous map, we have a pullback map $H_{T}^{*}\left(M_{2}\right) \rightarrow H_{T}^{*}\left(M_{1}\right)$. It is a ring homomorphism. In particular, we always have $H_{T}^{*}(\mathrm{pt}) \rightarrow H_{T}^{*}(M)$. Therefore $H_{T}^{*}(M)$ is a ring over $H_{T}^{*}(\mathrm{pt}) \cong \mathbb{C}\left[a_{1}, \ldots, a_{r}\right]$. We consider $H_{T}^{*}(M)$ as a coherent sheaf on $\operatorname{Spec}\left(\mathbb{C}\left[a_{1}, \ldots, a_{r}\right]\right)=\mathbb{C}^{r}$ in this way, and this view point is useful in the localization theorem below.

Suppose $T$ acts freely on $M$, and $M \rightarrow M / T$ is a principal $T$-bundle. Then $M_{V}$ is a fiber bundle over $M / T$ with fiber $V \backslash\{0\}$. Since the fiber has trivial cohomology groups, the spectral sequence for a fiber bundle gives us

$$
H_{T}^{i}(M) \cong H^{i}(M / T) .
$$

Moreover, $H_{T}^{>0}(\mathrm{pt})$ acts by 0 , as it is so on the $E^{2}$ term. In this case, $H_{T}^{i}(M)$ vanishes if $i$ is sufficiently large for a reasonable $M / T$.

Note that $M_{V} \rightarrow\left(\mathbb{P}^{N}\right)^{r}$ is a fiber bundle with fiber $M$. The restriction to a fiber gives a forgetful homomorphism

$$
H_{T}^{*}(M) \rightarrow H^{*}(M) .
$$

More generally, we have a restriction homomorphism

$$
H_{T}^{*}(M) \rightarrow H_{T^{\prime}}^{*}(M)
$$

for a subtorus $T^{\prime} \subset T$. Considering the case $M=\mathrm{pt}$, we find that we have an intrinsic description of (1.2):

$$
H_{T}^{*}(\mathrm{pt}) \cong \mathbb{C}[\operatorname{Lie} T],
$$

where $\mathbb{C}[\operatorname{Lie} T]$ is the ring of polynomial functions on Lie $T$. The homomorphism $H_{T}^{*}(\mathrm{pt})=\mathbb{C}[\operatorname{Lie} T] \rightarrow H_{T^{\prime}}^{*}(\mathrm{pt})=\mathbb{C}\left[\right.$ Lie $\left.T^{\prime}\right]$ is induced from the embedding Lie $T^{\prime} \rightarrow$ Lie $T$. 
If $E$ is a $T$-equivariant (complex) vector bundle over $M$, it induces a vector bundle $E_{V}=(V \backslash\{0\}) \times_{T} E$ over $M_{V}$. We define an equivariant Chern class $c_{i}(E)$ by $c_{i}\left(E_{V}\right)$. If $E$ is rank $r$, the top Chern class $c_{r}(E)$ is equal to the equivariant Euler class $e(E)$, defined in the same way.

If $M=$ pt, a $T$-equivariant vector bundle is nothing but a representation of $T$. The above $a_{i}$ in (1.2) is the equivariant first Chern class of the representation $T \rightarrow \mathbb{C}^{*}$, the projection to the $i^{\text {th }}$ factor. Then $c_{i}(E)$ is the $i^{\text {th }}$ elementary symmetric function of weights of $E$, regarded as a representation of $T$. Here weights are considered as linear functions Lie $T \rightarrow \mathbb{C}$. The equivariant Euler class $e(E)$ is the product of weights.

1(ii). Equivariant homology groups. In [24, Ch.8] we used both ordinary and Borel-Moore (or locally finite) homology groups to deal with Hilbert schemes of points on a noncompact surface $X$, like $X=$ $\mathbb{C}^{2}$. Here in the equivariant case, we mainly use Borel-Moore homology groups, since it fits better with convolution products.

Let $M$ be a $T$-variety as above. We define

$$
H_{i}^{T, l f}(M) \stackrel{\text { def. }}{=} H_{i+2 \operatorname{dim} V-2 \operatorname{dim} T}^{l f}\left(M_{V}\right) .
$$

It is independent of $V$ by the same argument as above, where we use $H_{i}^{l f}(V \backslash\{0\})=0$ for $2(\operatorname{dim} V-N-1)<i<2 \operatorname{dim} V$. Then $H_{*}^{T, l f}(M)$ is a module over $H_{T}^{*}(M)$ under the cap product. In particular, it is also a module over $H_{T}^{*}(\mathrm{pt})$.

If $T$ acts free on $M$ and $M \rightarrow M / T$ is a fiber bundle, we have

$$
H_{i}^{T, l f}(M) \cong H_{i-2 \operatorname{dim} T}^{l f}(M / T) .
$$

If $M$ is a smooth manifold of $\operatorname{dim} M=m$ with a smooth $T$-action, we define the equivariant fundamental class $[M]$ as $\left[M_{V}\right] \in H_{m+2 \operatorname{dim} V-2 \operatorname{dim} T}^{l f}\left(M_{V}\right)$. We have the Poincaré duality

$$
H_{T}^{i}(M) \cong H_{m-i}^{T, l f}(M) ; \quad c \mapsto c \cap[M]
$$

Even for an irreducible complex algebraic variety $M$ with a $T$-action, not necessarily smooth, its fundamental class $[M] \in H_{2 \operatorname{dim} M}^{T, l f}(M)$ is defined, as $\left[M_{V}\right]$ is defined. However the homomorphism $H_{T}^{i}(M) \rightarrow$ $H_{2 \operatorname{dim} M-i}^{T, l f}(M)$ may not be an isomorphism in general. Note also that

$$
H_{i}^{T, l f}(M)=0 \quad \text { if } i>2 \operatorname{dim} M .
$$

On the other hand, $H_{i}^{T, l f}(M)$ may be nonzero even for $i<0$.

If $f: M_{1} \rightarrow M_{2}$ is a proper $T$-equivariant map, we have the pushforward homomorphism $f_{*}: H_{*}^{T, l f}\left(M_{1}\right) \rightarrow H_{*}^{T, l f}\left(M_{2}\right)$. 
Considering the spectral sequence for the fiber bundle $M_{V} \rightarrow\left(\mathbb{P}^{N}\right)^{r}$, we have a forgetful homomorphism

$$
H_{i}^{T, l f}(M) \rightarrow H_{i}^{l f}(M) .
$$

We define the ordinary equivariant homology group $H_{i}^{T}(M)$ as the dual space to $H_{T}^{i}(M)$. This is enough for our purpose thanks to the universal coefficient theorem, as we only consider complex coefficients. We have $f_{*}: H_{i}^{T}\left(M_{1}\right) \rightarrow H_{i}^{T}\left(M_{2}\right)$ for a $T$-equivariant map, not necessarily proper. It is defined as the transpose of $f^{*}$. If $M$ is a smooth $T$-manifold, we have the cap product

$$
\cap: H_{i}^{T, l f}(M) \otimes H_{j}^{T}(M) \rightarrow H_{i+j-\operatorname{dim} M}^{T}(M)
$$

thanks to (1.5). We define the intersection pairing

$$
H_{i}^{T, l f}(M) \otimes H_{j}^{T}(M) \rightarrow H_{i+j-\operatorname{dim} M}^{T}(\mathrm{pt}) \cong H_{T}^{\operatorname{dim} M-i-j}(\mathrm{pt})
$$

as $a_{M *}(\bullet \cap \bullet)$, where $a_{M}: M \rightarrow$ pt. The second isomorphism is the Poincaré duality for pt.

We do not review further properties of equivariant Borel-Moore homology groups, which we will use implicitly in the next section. They are listed in $[24, \S 8.2]$ for nonequivariant Borel-Moore homology groups, and equivariant versions are simple consequences of nonequivariant ones applied to $M_{V}$.

1(iii). Localization theorem. In this subsection we explain the localization theorem in equivariant Borel-Moore homology groups, which relates the equivariant Borel-Moore homology of $M$ and that of the fixed point set $M^{T}$. In many situations, the fixed point set consists of a finite set, so the latter is just a direct sum of the equivariant cohomology of points (cf. (1.3)). Therefore the localization theorem is useful to say something on $H_{*}^{T, l f}(M)$.

A key point is that we view $H_{*}^{T}(M)$ as a module over $H_{T}^{*}(\mathrm{pt}) \cong$ $\mathbb{C}[$ Lie $T]$, or a sheaf on Lie $T$.

Recall that $H_{T}^{>0}(\mathrm{pt})$ acts trivially on $H_{*}^{T}(M)$ when $T$ acts freely on $M$, see (1.4). Therefore the support of $H_{*}^{T}(M)$ is 0 in Lie $T$. More generally, we have

Lemma 1.7. Suppose that the stabilizers of arbitrary points $x \in M$ is a fixed subgroup of $T^{\prime} \subset T$. Then the support of $H_{*}^{T}(M)$ is contained in $\operatorname{Lie}\left(T^{\prime}\right)$.

Proof. By the assumption, $M_{V}$ is a fiber bundle over $M /\left(T / T^{\prime}\right)$ with fiber $V \backslash\{0\} / T^{\prime}$. Then the action of $H_{T}^{*}(\mathrm{pt}) \cong \mathbb{C}[$ Lie $T]$ factors through $\mathbb{C}\left[\right.$ Lie $\left.T^{\prime}\right]$ on the $E^{2}$ term, and hence also on $H_{*}^{T}(M)$. 
Let $M^{T}$ be the fixed point set in $M$, and consider $H_{*}^{T}\left(M \backslash M^{T}\right)$. Since $M$ can be equivariantly embedded into a projective space, there are only finitely many stabilizers occur. We claim

$$
\operatorname{Supp} H_{*}^{T}\left(M \backslash M^{T}\right) \subset \bigcup_{x \in M \backslash M^{T}} \operatorname{Lie}(\operatorname{Stab}(x)) .
$$

We decompose $M \backslash M^{T}$ according to stabilizers into $\bigsqcup M_{\alpha}$. And we can order the index set $\{\alpha\}$ so that $M_{\leq \alpha}=\bigcup_{\beta: \beta \leq \alpha} M_{\beta}$ is closed in $M \backslash M^{T}$. We set $M_{<\alpha}=\bigcup_{\beta: \beta<\alpha} M_{\beta}$. From an exact sequence

$$
\cdots \rightarrow H_{i}^{T}\left(M_{<\alpha}\right) \rightarrow H_{i}^{T}\left(M_{\leq \alpha}\right) \rightarrow H_{i}^{T}\left(M_{\alpha}\right) \rightarrow H_{i-1}^{T}\left(M_{<\alpha}\right) \rightarrow \cdots
$$

and Lemma 1.7 applied to $M_{\alpha}$, we deduce that the support of $H_{*}^{T}\left(M_{\leq \alpha}\right)$ is contained in the right hand side of (1.8) by an induction on $\alpha$. Therefore using the exact sequence for $H_{*}^{T}(M), H_{*}^{T}\left(M^{T}\right), H_{*}^{T}\left(M \backslash M^{T}\right)$, we get

Theorem 1.9. Let $i: M^{T} \rightarrow M$ be the inclusion of the fixed point set. Then the kernel and cokernel of the homomorphism

$$
i_{*}: H_{*}^{T}\left(M^{T}\right) \rightarrow H_{*}^{T}(M)
$$

are supported in $\bigcup_{x \in M \backslash M^{T}} \operatorname{Lie}(\operatorname{Stab}(x))$.

Corollary 1.10. Let $\operatorname{Frac}\left(H_{T}^{*}(\mathrm{pt})\right)$ be the fractional field of $H_{T}^{*}(\mathrm{pt})$ and let $H_{*}^{T}(M)_{\mathbb{F}}=H_{*}^{T}(M) \otimes_{H_{T}^{*}(\mathrm{pt})} \operatorname{Frac}\left(H_{T}^{*}(\mathrm{pt})\right)$, and similarly for $H_{*}^{T}\left(M^{T}\right)_{\mathbb{F}}$. Then

$$
i_{*}: H_{*}^{T}\left(M^{T}\right)_{\mathbb{F}} \rightarrow H_{*}^{T}(M)_{\mathbb{F}}
$$

is an isomorphism.

1(iv). Fixed point formula. When $M$ is nonsingular, we have the Poincaré duality (1.5). Therefore we have two isomorphisms $i_{*}: H_{*}^{T}\left(M^{T}\right)_{\mathbb{F}} \rightarrow$ $H_{*}^{T}(M)_{\mathbb{F}}$ and $i^{*}: H_{*}^{T}(M)_{\mathbb{F}} \rightarrow H_{*}^{T}\left(M^{T}\right)_{\mathbb{F}}$. Having both are are very useful, as $i^{*} i_{*}$ can be explicitly written down. This leads us to the fixed point formula.

Let $M^{T}=\bigsqcup M_{\alpha}$ be the decomposition to connected components. Each $M_{\alpha}$ is a nonsingular subvariety of $M$. Let $N_{\alpha}$ denote its normal bundle. We have

$$
H_{*}^{T}\left(M^{T}\right)=\bigoplus_{\alpha} H_{*}^{T}\left(M_{\alpha}\right)
$$

Lemma 1.11. Let $i_{\alpha}$ be the inclusion of $M_{\alpha}$ into $M$. Then $i_{\alpha}^{*} i_{\alpha *}$ is given by the cap product $e\left(N_{\alpha}\right) \cap \bullet$ of the equivariant Euler class of $N_{\alpha}$. 
Proof. In the neighborhood of $M_{\alpha}, M$ is isomorphic to a neighborhood of the 0 -section of $N_{\alpha}$. Therefore we may replace $i_{\alpha}: M_{\alpha} \rightarrow M$ by the inclusion $M_{\alpha} \rightarrow N_{\alpha}$ of the 0 -section. We then obtain the assertion by the Thom isomorphism.

Lemma 1.12. $e\left(N_{\alpha}\right) \cap \bullet$ is invertible in $H_{*}^{T}\left(M_{\alpha}\right)_{\mathbb{F}}$.

Proof. Since $T$ acts trivially on $M_{\alpha}$, we have $H_{T}^{*}\left(M_{\alpha}\right)=H^{*}\left(M_{\alpha}\right) \otimes$ $H_{T}^{*}(\mathrm{pt})$. Note that $H^{>0}\left(M_{\alpha}\right) \otimes H_{T}^{*}(\mathrm{pt})$ is nilpotent as $H^{>2 \operatorname{dim} M_{\alpha}}\left(M_{\alpha}\right)=$ 0 . Therefore it is enough to check that $H^{0}\left(M_{\alpha}\right) \otimes H_{T}^{*}(\mathrm{pt})$ part of $e\left(N_{\alpha}\right)$ is nonzero. It is enough to study it after restricting $N_{\alpha}$ to a point $x \in M_{\alpha}$.

For $x \in M_{\alpha}, T_{x} M$ is a $T$-module so that $T_{x} M_{\alpha}$ is its weight 0 subspace. Therefore $\left.e\left(N_{\alpha}\right)\right|_{x}$ is the product of nonzero weights of $T_{x} M_{\alpha}$. Therefore it is nonzero.

Let us denote the inverse of $e\left(N_{\alpha}\right) \cap \bullet$ by $\frac{1}{e\left(N_{\alpha}\right)}$.

Theorem 1.13. Suppose $M$ is nonsingular. Then the inverse of $i_{*}$ in Corollary 1.10 is given by

$$
\sum_{\alpha} \frac{1}{e\left(N_{\alpha}\right)} i_{\alpha}^{*}
$$

Proof. Note that we have decomposition $H_{*}^{T}\left(M^{T}\right)=\bigoplus H_{*}^{T}\left(M_{\alpha}\right)$, and hence we have $i_{*}=\sum i_{\alpha *}$. By Lemmas 1.11, 1.12, $e\left(N_{\alpha}\right)^{-1} i_{\alpha}^{*} i_{\alpha *}$ is the identity operator on $H_{*}^{T}\left(M_{\alpha}\right)_{\mathbb{F}}$. Therefore we have the assertion.

We now arrive at Atiyah-Bott-Berline-Vergne fixed point formula.

Theorem 1.14. Assume $M$ is proper and nonsingular. Let $a: M \rightarrow$ pt, $a_{\alpha}: M_{\alpha} \rightarrow$ pt. Then we have an equality in $H_{*}^{T}(\mathrm{pt})_{\mathbb{F}} \cong \mathbb{C}\left(a_{1}, \ldots, a_{r}\right)$ for $\omega \in H_{*}^{T}(M)$ :

$$
a_{*}(\omega)=\sum_{\alpha} a_{\alpha *}\left(\frac{1}{e\left(N_{\alpha}\right)} i_{\alpha}^{*} \omega\right) .
$$

Proof. We have

$$
a_{*}(\omega)=a_{*} i_{*} i_{*}^{-1}(\omega)=\sum_{\alpha} a_{*} i_{\alpha *} \frac{1}{e\left(N_{\alpha}\right)} i_{\alpha}^{*}(\omega) .
$$

Since $a_{\alpha}=a \circ i_{\alpha}$, we get the assertion. 


\section{Equivariant homology groups of Hilbert schemes of POINTS}

We explain the construction of the Fock space representation of the Heisenberg algebra in [24, Ch. 8] in equivariant homology groups in this section. It was first noticed by Vasserot [27] that the arguments in [24, Ch. 8] work in the equivariant setting, and such a generalization is quite useful.

2(i). Heisenberg algebra. Let $X=\mathbb{C}^{2}$ with the linear coordinate system $(z, \xi)$. Two dimensional torus $T$ acts on $X$ by $\left(t_{1}, t_{2}\right) \cdot(z, \xi)=$ $\left(t_{1} z, t_{2} \xi\right)$.

Let $X^{[n]}$ denote the Hilbert scheme of $n$ points in $X$. Let $\pi: X^{[n]} \rightarrow$ $S^{n} X$ be the Hilbert-Chow morphism. We have induced $T$-actions on $X^{[n]}$ and $S^{n} X$ so that $\pi$ is equivariant.

Recall $([24,(8.9)])$ that we considered a subvariety $P[i] \subset \bigsqcup_{n} X^{[n]} \times$ $X^{[n-i]} \times X$ defined by

$$
P[i] \stackrel{\text { def. }}{=}\left\{\left(I_{1}, I_{2}, x\right) \mid I_{1} \subset I_{2}, \operatorname{Supp}\left(I_{2} / I_{1}\right)=\{x\}\right\}
$$

for $i>0$. Let us omit $\bigsqcup_{n}$ for brevity hereafter.

The projections $q_{1}: P[i] \rightarrow X^{[n]}, q_{2}: P[i] \rightarrow X^{[n-i]} \times X$ are proper. Therefore convolution operators

$$
\begin{aligned}
& H_{*}^{T, l f}\left(X^{[n-i]} \times X\right) \rightarrow H_{*}^{T, l f}\left(X^{[n]}\right) ; \bullet \mapsto q_{1 *}\left(q_{2}^{*}(\bullet) \cap[P[i]]\right), \\
& H_{*}^{T, l f}\left(X^{[n]}\right) \rightarrow H_{*}^{T, l f}\left(X^{[n-i]} \times X\right) ; \bullet \mapsto(-1)^{i} q_{2 *}\left(q_{1}^{*}(\bullet) \cap[P[i]]\right)
\end{aligned}
$$

are well-defined. The sign $(-1)^{i}$ is introduced so that the commutation relation below is simplified. We take the direct sum of homology groups over $n$ later, keeping the same notation.

Take an equivariant class $\beta \in H_{*}^{T, l f}(X)$. We replace $\bullet$ by $\bullet \otimes \beta \in$ $H_{*}^{T, l f}\left(X^{[n-i]} \times X\right) \cong H_{*}^{T, l f}\left(X^{[n-i]}\right) \otimes H_{*}^{T, l f}(X)$ in the first operator in (2.2). It is considered as an operator $H_{*}^{T, l f}\left(X^{[n-i]}\right) \rightarrow H_{*}^{T, l f}\left(X^{[n]}\right)$. Let us denote it by $P_{-i}(\beta)$. It was denoted by $P_{\beta}[-i]$ in [24].

On the other hand, for the second operator in (2.2), we take the intersection pairing with $\alpha \in H_{*}^{T}(X)$ via $H_{*}^{T, l f}\left(X^{[n-i]} \times X\right) \cong H_{*}^{T, l f}\left(X^{[n-i]}\right) \otimes$ $H_{*}^{T, l f}(X)$. We obtain an operator $H_{*}^{T, l f}\left(X^{[n]}\right) \rightarrow H_{*}^{T, l f}\left(X^{[n-i]}\right)$, which is denoted by $P_{i}(\alpha)$.

These operators $P_{i}(\alpha), P_{-i}(\beta)$ are the same as ones in [24, Ch.8] though the current explanation is slightly different. 
We can replace $H_{*}^{T}$ by the equivariant Borel-Moore homology group $H_{*}^{T, l f}$ in $(2.2)$. Then

$$
\begin{aligned}
& P_{i}(\alpha): H_{*}^{T, l f}\left(X^{[n]}\right) \rightarrow H_{*}^{T, l f}\left(X^{[n-i]}\right), \\
& P_{-i}(\beta): H_{*}^{T, l f}\left(X^{[n-i]}\right) \rightarrow H_{*}^{T, l f}\left(X^{[n]}\right)
\end{aligned}
$$

are well-defined for $\alpha \in H_{*}^{T}(X), \beta \in H_{*}^{T, l f}(X)$. Note that homology groups containing $\alpha, \beta$ are swapped from the above case.

We have a perfect pairing

$$
\langle,\rangle: H_{*}^{T, l f}\left(X^{[n]}\right) \otimes H_{*}^{T}\left(X^{[n]}\right) \rightarrow H_{*}^{T}(\mathrm{pt}) ; c \otimes c^{\prime} \mapsto(-1)^{n} a_{*}\left(c \cap c^{\prime}\right)
$$

by the Poincaré duality. Here $a: X^{[n]} \rightarrow$ pt. The transpose of $P_{i}(\alpha)$ is equal to $P_{-i}(\alpha)$.

The operators are linear over $H_{T}^{*}(\mathrm{pt})$ from the construction: $P_{i}(\alpha) f=$ $f P_{i}(\alpha)$, etc. for $f \in H_{T}^{*}(\mathrm{pt})$. It is $H_{T}^{*}(\mathrm{pt})$-linear on $\alpha: P_{i}(f \alpha)=f P_{i}(\alpha)$, etc.

Note that $H_{*}^{T}(X) \cong H_{T}^{*}(\mathrm{pt})[0], H_{*}^{T, l f}(X) \cong H_{T}^{*}(\mathrm{pt})[X]$. Therefore it is enough to consider the cases $\alpha, \beta=[0]$ and $[X]$.

Then the following commutation relation of the Heisenberg algebra holds:

$$
\left[P_{i}(\alpha), P_{j}(\beta)\right]=i \delta_{i+j, 0}\langle\alpha, \beta\rangle \mathrm{id} .
$$

When the right hand side is nonzero, i.e., $i+j=0$, one of $\alpha$ or $\beta$ is in $H_{*}^{T}(X)$ and the other is in $H_{*}^{T, l f}(X)$. Therefore

$$
\langle\alpha, \beta\rangle=-a_{X *}(\alpha \cap \beta) \in H_{T}^{*}(\mathrm{pt})
$$

is well-defined, where $a_{X}: X \rightarrow$ pt. We take the same sign convention as above, understanding $X^{[1]}=X$, i.e., it is $(-1)$ times the intersection pairing.

The proof of (2.5) for nonequivariant homology groups in [24, Ch.8] works also for the equivariant case. Let us briefly explain checkpoints. When $i+j \neq 0$, the right hand side of (2.5) vanishes. In these cases, we study set-theoretical intersections of cycles, check that they are transversal intersections generically and non-generic parts do not contribute to the computation by dimension reason. The last reasoning works in the equivariant case thanks to (1.6).

When $i+j=0$, the argument in [24] works before the sentence "If $\operatorname{deg} \alpha+\operatorname{deg} \beta<4$, then $\alpha \cap \beta=0$ " in the middle of p.102. This is not true for the equivariant case, as $H_{<0}^{T}(X)$ is nonzero. So let us go back a little and see what is actually proved there. We consider

$$
L \stackrel{\text { def. }}{=}\left\{\left(I_{1}, I_{3}, x\right) \in X^{[n]} \times X^{[n]} \times X \mid I_{1}=I_{3} \text { outside } x\right\} .
$$


Then $L$ has $n$ irreducible components $L_{1}, \ldots, L_{n}$ with $\operatorname{dim}_{\mathbb{C}} L_{i}=$ $2 n$, and $(2 n+2)$-dimensional irreducible component $\Delta_{X^{[n]}} \times X$, and other irreducible components (if exists) have lower dimension (see [24, Lemma 8.32]). We have the projection $\Pi^{\prime \prime}: L \rightarrow X$ to the third factor. Then by the same argument as the nonequivariant case, we show that there is a class $\mathscr{R}$ in $H_{4 n+4}^{T, l f}(L)$ (which is $p_{134 *} \iota_{*}^{\prime \prime} R^{\prime \prime}-p_{134 *} \iota_{*}^{\prime \prime \prime} R^{\prime \prime \prime}$ in the notation [24, p.101]) such that the left hand side of (2.5) is given by the correspondence

$$
p_{13 *}\left(\Pi^{\prime \prime *}(\alpha \cap \beta) \cap \mathscr{R}\right) \in H_{4 n+\operatorname{deg} \alpha+\operatorname{deg} \beta-4}^{T, l f}\left(X^{[n]} \times_{S^{n} X} X^{[n]}\right),
$$

where $p_{13}: L \rightarrow X^{[n]} \times{ }_{S^{n} X} X^{[n]}$ is the projection $\left(I_{1}, I_{3}, x\right) \mapsto\left(I_{1}, I_{3}\right)$. By dimension reason, we see that $\mathscr{R}$ comes from a class in $H_{4 n+4}^{T, l f}\left(\Delta_{X^{[n]}} \times\right.$ $X)$. Since it is of top degree, it must be $c_{i, n}\left[\Delta_{X}^{[n]} \times X\right]$, where the multiple constant $c_{i, n}$ is independent of equivariant variables, i.e., a complex number. Therefore

$$
p_{13 *}\left(\Pi^{\prime \prime *}(\alpha \cap \beta) \cap \mathscr{R}\right)=c_{i, n}\langle\alpha, \beta\rangle\left[\Delta_{X}{ }^{[n]}\right] .
$$

Now the only task is to determine $c_{i, n}$. Since $c_{i, n}$ is a complex number, we can take nonequivariant limit: it is the same for equivariant and nonequivariant cases. Therefore we conclude $c_{i, n}=i$.

2(ii). Torus fixed points. For a later purpose, let us recall fixed points with respect to the two dimensional torus $T$. They are parametrized by partitions $\lambda$ of $n$, or equivalently Young diagrams $D$ with $n$ boxes. (See [24, Ch. 5 and 6].) We keep our convention in [24]: for $\lambda=$ $\left(\lambda_{1}, \lambda_{2}, \ldots\right)$, the corresponding torus fixed point is the monomial ideal

$$
I_{\lambda}=\left(\xi^{\lambda_{1}}, z \xi^{\lambda_{2}}, \ldots, z^{i-1} \xi^{\lambda_{i}}, \ldots\right) .
$$

If we put the monomial $z^{i-1} \xi^{j-1}$ in the box at the intersection of the $i^{\text {th }}$ column and the $j^{\text {th }}$ row, $I_{\lambda}$ is generated by monomials which sit outside of $D$. See Figure 1.

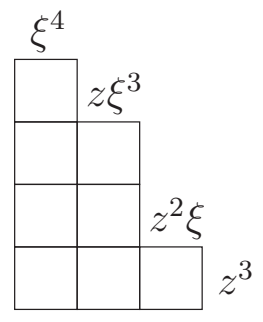

FiguRE 1. Young diagram and an ideal 
2(iii). Symmetric products of the $x$-axis. The constant $c_{i, n}$ (in fact, it is easy to see that it is independent of $n$ by a similar argument as above) was determined by studying symmetric products of an embedded curve $C$ in $X$ in the nonequivariant case [24, Ch.9]. We shall briefly review the study here, emphasizing that the argument goes through even when $C$ and $X$ are noncompact. We shall use the construction later.

Let $C$ denote the z-axis, i.e., $C=\{\xi=0\}$. Let $L^{*} C$ denote the subvariety consisting of $I \in \bigsqcup_{n} X^{[n]}$ such that $\mathbb{C}[z, \xi] / I$ is contained in $C$. In other words, it is the union $\bigsqcup_{n} \pi^{-1}\left(S^{n} C\right)$ of inverse images of the symmetric products $S^{n} C$ under the Hilbert-Chow morphism.

By [24, Ch.7] it has irreducible components $L^{\lambda} C$ parametrized by partitions $\lambda$. Moreover, it is a lagrangian subvariety in $X^{[n]}$ with $n=$ $|\lambda|$. We recall two descriptions of irreducible component $L^{\lambda} C$.

The first one is by the $\mathbb{C}^{*}$-action given by $t \cdot(z, \xi) \mapsto(z, t \xi)$. Then $I \in \mathbb{C}^{*}$ is fixed by $\mathbb{C}^{*}$ if and only if it is of a form

$$
I=I_{D_{1}, z_{1}} \cap \cdots \cap I_{D_{k}, z_{k}}
$$

where $z_{k} \in C, D_{i}$ is a Young diagram and $I_{D_{i}, z_{i}}$ is the ideal generated by monomials in $\left(z-z_{i}\right)$ and $\xi$ which sit outside of $D_{i}$ as in $\S 2(i i)$. The diagram $D_{i}$ and the point $z_{i}$ is uniquely determined by $I$. Let $D$ be the union of all $D_{i}$, reordering columns so that it is a Young diagram. Let $\lambda$ be the corresponding partition. Then $S^{\lambda} C$ consisting of fixed points $I$ with $D=D_{1} \sqcup D_{2} \sqcup \cdots$ is a connected component of $\left(X^{[n]}\right)^{\mathbb{C}^{*}}$, and any connected component is of this form.

We have

$$
L^{*} C=\bigsqcup_{n}\left\{I \in X^{[n]} \mid \lim _{t \rightarrow \infty} t \cdot I \text { exists }\right\}
$$

which decomposes according to the limit as $L^{*} C=\bigsqcup W_{\lambda}^{-}$with

$$
W_{\lambda}^{-} \stackrel{\text { def. }}{=}\left\{I \in L^{*} C \mid \lim _{t \rightarrow \infty} t \cdot I \in S^{\lambda} C\right\} .
$$

Each $W_{\lambda}^{-}$is a locally closed lagrangian subvariety in $X^{[n]}$ and we define

$$
L^{\lambda} C \stackrel{\text { def. }}{=} \text { Closure of } W_{\lambda}^{-} \text {. }
$$

For example, $\lambda=\left(1^{n}\right), L^{\lambda} C$ is isomorphic to the symmetric product $S^{n} C$ of $C$, embedded into $X^{[n]}$ via the natural morphism $S^{n} C \cong C^{[n]} \rightarrow$ $X^{[n]}$. This is clearly a lagrangian subvariety in $X^{[n]}$ since the symplectic form is an extension of one on the open subset $\pi^{-1}\left(S_{\left(1^{n}\right)}^{n} X\right)$. The opposite extreme is $L^{(n)} C$, which consists of ideals $I$ such that $\mathbb{C}[z, \xi] / I$ is supported at a single point in $C$. The lagrangian property is less clear, and follows from the Morse theoretic argument in [24, Ch.7]. 
The second description is by the Hilbert-Chow morphism $\pi: X^{[n]} \rightarrow$ $S^{n} X$. Recall we have $L^{*} C=\pi^{-1}\left(S^{n} C\right)$. Note that $S^{n} C$ has the natural stratification $S^{n} C=\bigsqcup S_{\lambda}^{n} C$, where

$$
S_{\lambda}^{n} C=\left\{\sum_{i} \lambda_{i}\left[x_{i}\right] \in S^{n} C \mid x_{i} \neq x_{j} \text { for } i \neq j\right\} .
$$

We decompose $L^{*} C$ accordingly

$$
L^{*} C=\bigsqcup \pi^{-1}\left(S_{\lambda}^{n} C\right)
$$

Each $\pi^{-1}\left(S_{\lambda}^{n} C\right)$ is a locally closed $n$-dimensional irreducible subvariety in $L^{*} C\left(\cap X^{[n]}\right)$, where the irreducibility follows from that of punctual Hilbert schemes $\pi^{-1}(m[p])$ ([24, Th. 5.12]). Hence its closure is an irreducible component.

Proposition 2.17. $L^{\lambda} C$ is the closure of $\pi^{-1}\left(S_{\lambda}^{n} C\right)$.

Proof. It is enough to note that the above component $S^{\lambda} C$ of $\left(X^{[n]}\right)^{\mathbb{C}^{*}}$ has an open locus consisting of $I=I_{D_{1}, z_{1}} \cap \cdots \cap I_{D_{k}, z_{k}}$ such that all $D_{i}$ 's have only single column.

There is the third description, which was not given in [24]: Let us consider the hyperbolic $\mathbb{C}^{*}$-action $t *(z, \xi)=\left(t^{-1} z, t \xi\right)$. Then we claim that $I \in \bigsqcup_{n} X^{[n]}$ has limit when $t \rightarrow \infty$ if and only if $I \in L^{*} C$. If we replace $X^{[n]}$ by the symmetric product $S^{n} X$, the corresponding assertion is obvious. A point $\sigma \in S^{n} X$ has limit when $t \rightarrow \infty$ if and only if $\sigma \in S^{n} C$. Since $\pi$ is proper, the assertion follows. Then $L^{*} C$ decomposes according to the decomposition of $\left(X^{[n]}\right)^{\mathbb{C}^{*}}$ as in (2.13), where the $\mathbb{C}^{*}$-action is replaced by the hyperbolic one. Let us first note that $\left(X^{[n]}\right)^{\mathbb{C}^{*}}=\left(X^{[n]}\right)^{T}$. This follows, for example, from the $T$ character formula of the tangent space at $I_{\lambda} \in\left(X^{[n]}\right)^{T}$ reviewed in Proposition 3.19 below. Put $t_{1}=t_{2}^{-1}=t$. We see that $T_{I_{\lambda}} X^{[n]}$ has a trivial weight zero space. It means that $\left(X^{[n]}\right)^{\mathbb{C}^{*}}$ cannot be larger than $\left(X^{[n]}\right)^{T}$.

\section{Proposition 2.18.}

$$
W_{\lambda}^{-}=\left\{I \in L^{*} C \mid \lim _{t \rightarrow \infty} t * I=I_{\lambda}\right\} .
$$

Proof. Since both left and right hand sides are $T$-invariant locally closed submanifolds, it is enough to prove the equality in a neighborhood of the fixed point $I_{\lambda}$.

The tangent space of $W_{\lambda}^{-}$at $I_{\lambda}$ is equal to the sum of nonpositive weight spaces in $T_{I_{\lambda}} X^{[n]}$ with respect to the $\mathbb{C}^{*}$-action given by $t$. $(z, \xi)=(z, t \xi)$. Similarly, the tangent space of the right hand side is the 
sum of negative weight spaces with respect to the hyperbolic $\mathbb{C}^{*}$-action $t *(z, \xi)=\left(t^{-1} z, t \xi\right)$. Looking at the formula in Proposition 3.19 below, one finds that both spaces are the same space. In fact, it corresponds to $\sum t_{1}^{l(s)+1} t_{2}^{-a(s)}$. (One can also check that it is a lagrangian subspace in $T_{I_{\lambda}} X^{[n]}$, as the symplectic form is of weight $t_{1} t_{2}$.)

There is no nontrivial $T$-homomorphism from any symmetric power of $T_{I_{\lambda}} W_{\lambda}^{-}$to the sum of complementary weight spaces, and hence a $T$-invariant submanifold with the tangent space $T_{I_{\lambda}} W_{\lambda}^{-}$is unique, as in the proof of the existence of Białynicki-Birula decomposition (see [5, Th. 2.2]). Therefore two submanifolds are equal.

Let us consider the top degree Borel-Moore homology group of $L^{*} C$ :

$$
H_{\mathrm{top}}^{l f}\left(L^{*} C\right)=\bigoplus_{n} H_{2 n}^{l f}\left(L^{*} C \cap X^{[n]}\right) .
$$

This is a vector space with a base $\left\{\left[L^{\lambda} C\right]\right\}$. The creation operator $P_{-i}(\beta)$ acts on $H_{\text {top }}^{l f}\left(L^{*} C\right)$ for $\beta=[C] \in H_{2}^{l f}(C)$. On the other hand, for the annihilation operator $P_{i}(\alpha)$, we take $\alpha=[y$-axis $]$. Then the intersection pairing of $\alpha$ and $\beta$ is well-defined (in fact, it is 1), as $\{x$-axis $\} \cap\{y$-axis $\}$ is a single point in $X$, and $P_{i}(\alpha)$ is a well-defined operator on $H_{\text {top }}^{l f}\left(L^{*} C\right)$. The top degree is preserved by the convolution product, as $P[i]$ in (2.1) is middle dimensional in $X^{[n]} \times X^{[n-i]} \times X$, and $\alpha, \beta$ are so in $X$.

We have a linear map from the ring $\Lambda$ of symmetric functions to $H_{\text {top }}^{l f}\left(L^{*} C\right)$ by

$$
p_{\lambda}=p_{\lambda_{1}} p_{\lambda_{2}} \cdots \mapsto P_{-\lambda_{1}}(\beta) P_{-\lambda_{2}}(\beta) \cdots 1,
$$

for $\lambda=\left(\lambda_{1}, \lambda_{2}, \ldots\right)$. From the Heisenberg algebra relation (2.5), it is injective. (For this we only need that the constant $c_{i, n}$ is nonzero, which is a consequence of the Poincaré duality.) Since $\operatorname{dim} H_{\mathrm{top}}^{l f}\left(L^{*} C \cap X^{[n]}\right)$ is equal to the number of partitions of $n$, it is an isomorphism.

In order to determine the coefficient $i$ in (2.5), it is enough to compute it in the current situation. A key result is

Proposition 2.21. Under the isomorphism $\Lambda \cong H_{\mathrm{top}}^{l f}\left(L^{*} C\right)$, the class $\left[L^{\lambda} C\right]$ corresponds to the monomial symmetric function $m_{\lambda}$.

This result together with the formula

$$
P_{i}(\alpha)\left[L^{\left(1^{n}\right)} C\right]=\left[L^{\left(1^{n-i}\right)} C\right]
$$

(see [24, Lemma 9.21]) determines the coefficient. 


\section{JACK SYMMETRIC FUNCTIONS AND TORUS FIXED POINTS}

The goal of this section is to give a geometric realization of Jack symmetric functions in the $T$-equivariant homology groups of Hilbert schemes of points in $X=\mathbb{C}^{2}$.

The result presented here was stated and proved by Li-Qin-Wang [17]. Both the framework and the proof were based on an earlier work by Vasserot [27], who considered Schur functions and the $\mathbb{C}^{*}$ equivariant homology groups, instead of $T$-equivariant ones. Vasserot's work, in turn, was motivated by author's unpublished paper [22], where Jack symmetric functions were considered in a different setting, i.e., the case when $X$ is the total space of a line bundle over a Riemann surface.

Here we present the proof in [17], as well as materials in [22] used there.

The result here could be viewed as a homological version of Haiman's result [12], which relates Macdonald polynomials to $T$-equivariant $K$ theory of Hilbert schemes of points in $X$. However there is a big difference: we relate $H_{*}^{T}\left(X^{[n]}\right)$ with the ring of symmetric functions via the Heisenberg representation in the previous section. On the other hand, Haiman relates them via the Procesi bundle, a rank $n$ ! vector bundle over $X^{[n]}$. The definition of the Procesi bundle is rather involved, and will not be presented here. Let us also remark that the Procesi bundle is essential to prove the positivity conjecture for Macdonald polynomials, and we do not have a counter part in our theory.

3(i). Jack symmetric functions. Let us briefly recall the definition of Jack symmetric functions in [19, VI.10]. Our notation follows [19] except that the parameter $\alpha$ is replaced by $\boldsymbol{k}$ here.

Let $\boldsymbol{k}$ be an indeterminate. We define an inner product on the ring $\Lambda_{\mathbb{Q}(\boldsymbol{k})}$ of symmetric functions with coefficients in $\mathbb{Q}(\boldsymbol{k})$ by

$$
\left\langle p_{\lambda}, p_{\mu}\right\rangle=\delta_{\lambda \mu} \boldsymbol{k}^{l(\lambda)} z_{\lambda},
$$

where $l(\lambda)$ is the length of a partition $\lambda$ and $z_{\lambda}=\prod k^{m_{k}} m_{k}$ ! for $\lambda=$ $\left(1^{m_{1}} 2^{m_{2}} \cdots\right)$.

Let $\geq$ denote the dominance order on partitions, i.e., $\lambda \geq \mu$ if and only if $|\lambda|=|\mu|$ and

$$
\lambda_{1}+\cdots+\lambda_{i} \geq \mu_{1}+\cdots+\mu_{i} \text { for all } i .
$$

If $\lambda^{\prime}, \mu^{\prime}$ denote conjugate partitions of $\lambda, \mu$ respectively, we have

$$
\lambda \geq \mu \Longleftrightarrow \mu^{\prime} \geq \lambda^{\prime}
$$

See [19, I.(1.11)] for the proof. 
Let $m_{\lambda}$ be the monomial symmetric function. A Jack symmetric function $P_{\lambda}^{(k)}$ is characterized by the following two properties

$$
\begin{aligned}
& P_{\lambda}^{(\boldsymbol{k})}=m_{\lambda}+\sum_{\mu<\lambda} u_{\lambda \mu}^{(\boldsymbol{k})} m_{\mu} \quad \text { for } u_{\lambda \mu}^{(\boldsymbol{k})} \in \mathbb{Q}(\boldsymbol{k}), \\
& \left\langle P_{\lambda}^{(\boldsymbol{k})}, P_{\mu}^{(\boldsymbol{k})}\right\rangle=0 \quad \text { if } \lambda \neq \mu .
\end{aligned}
$$

Let $\succeq$ be a total order refining the dominance order. Then $\left\{P_{\lambda}^{(\boldsymbol{k})}\right\}$ is obtained from the base $\left\{m_{\lambda}\right\}$ by Gram-Schmidt orthogonalization with respect to $\succeq$. Hence the uniqueness of $P_{\lambda}^{(\boldsymbol{k})}$ follows. The existence is obvious if we replace $\geq$ by $\succeq$ in (3.3), and this difference of orders is a nontrivial part in the theory of Jack symmetric functions. We briefly recall the proof in [19] in the next subsection.

When $\boldsymbol{k}=1$, the inner product (3.1) is the standard one. The above two properties are satisfied by Schur functions $s_{\lambda}$.

3(ii). Hamiltonian. For a positive integer $N$, let us consider the ring $\Lambda_{N, \mathbb{Q}(\boldsymbol{k})}$ of symmetric functions in $N$-variables $x_{1}, \ldots, x_{N}$ with $\mathbb{Q}(\boldsymbol{k})$ coefficients. (Note that $N, \boldsymbol{k}$ correspond to $n, \alpha$ in [19].)

Let $X$ be an indeterminate and let

$$
D_{N} \stackrel{\text { def. }}{=} a_{\delta}(x)^{-1} \sum_{w \in S_{N}} \varepsilon(w) x^{w \delta} \prod_{i=1}^{N}\left(X+(w \delta)_{i}+\boldsymbol{k} x_{i} \frac{\partial}{\partial x_{i}}\right),
$$

where $\delta=(N-1, N-2, \ldots, 0), a_{\delta}(x)=\prod_{i<j}\left(x_{i}-x_{j}\right)$ is the Vandermonde determinant, $\varepsilon(w)= \pm 1$ is the sign of $w \in S_{N}$, and $(w \delta)_{i}$ is the $i^{\text {th }}$ component of $w \delta$.

For $r=0,1, \ldots, N$ let $D_{N}^{r}$ denote the coefficient of $X^{N-r}$ in $D_{N}$ :

$$
D_{N}=\sum_{r=0}^{N} X^{N-r} D_{N}^{r}
$$

By [19, VI, §3. Ex.3(a)] we have

$$
D_{N} m_{\lambda}(x)=\sum_{w \in S_{N}} \prod_{i=1}^{N}\left(X+N-i+\boldsymbol{k}(w \delta)_{i}\right) s_{w \lambda}(x),
$$

where

$$
s_{w \lambda}(x)=\frac{a_{\delta+w \lambda}(x)}{a_{\delta}(x)}=\frac{1}{a_{\delta}(x)} \sum_{w_{1} \in S_{N}} \varepsilon\left(w_{1}\right) x^{w_{1}(w \lambda+\delta)} .
$$

(There is a typo in [19, VI, §3, Ex.3(a)]. In the formula (a), $\lambda_{i}$ should read $\beta_{i}$ or $(w \lambda)_{i}$ in the current notation.) Since $s_{w \lambda}(x)$ is either zero or is equal to $\pm s_{\mu}$ for some partition $\mu<\lambda$ (unless $w=1$ ), and since 
the transition matrix between $\left\{s_{\lambda}\right\}$ and $\left\{m_{\lambda}\right\}$ is upper triangular with 1 on diagonal, we get

$$
D_{N} m_{\lambda}(x)=\sum_{\mu \leq \lambda} c_{\lambda \mu}(X ; \boldsymbol{k}) m_{\mu}(x)
$$

with $c_{\lambda \mu} \in \mathbb{Z}[X, \boldsymbol{k}]$. See $\left[19, \mathrm{VI}, \S 4\right.$, Ex.4]. Here $m_{\lambda}(x)$ and $s_{\lambda}(x)$ are zero if $l(\lambda)>N$, and $\left\{m_{\lambda}(x)\right\}_{l(\lambda) \leq N},\left\{s_{\lambda}(x)\right\}_{l(\lambda) \leq N}$ are bases of $\Lambda_{N}$. The diagonal entries are given by

$$
c_{\lambda \lambda}(X ; \boldsymbol{k})=\prod_{i=1}^{N}\left(X+N-i+\boldsymbol{k} \lambda_{i}\right) .
$$

When we set $\boldsymbol{k}$ a positive real number, $N-i+\boldsymbol{k} \lambda_{i}$ is strictly decreasing in $i$. Therefore $c_{\lambda \lambda} \neq c_{\mu \mu}$ for $\lambda \neq \mu$. Therefore there exists $P_{\lambda}^{(\boldsymbol{k})}$ of the form (3.3) such that

$$
D_{N} P_{\lambda}^{(\boldsymbol{k})}=c_{\lambda \lambda}(X ; \boldsymbol{k}) P_{\lambda}^{(\boldsymbol{k})} .
$$

Moreover $D_{N}$ is self-adjoint with respect to the finitely many variable version of the inner product (3.1) [19, VI, §3, Ex.3(b)]. Therefore we deduce (3.4). It means that $\left\{P_{\lambda}^{(\boldsymbol{k})}\right\}$ is obtained from the base $\left\{m_{\lambda}\right\}$ by Gram-Schmidt orthogonalization with respect to any total order $\succeq$ compatible with $\geq$.

The ring $\Lambda_{\mathbb{Q}(\boldsymbol{k})}$ is defined as the direct sum of projective limit

$$
\Lambda_{\mathbb{Q}(\boldsymbol{k})}=\bigoplus{\underset{\leftarrow}{\lim }}_{\lim _{N, \mathbb{Q}(\boldsymbol{k})}},
$$

where $\Lambda_{N, \mathbb{Q}(\boldsymbol{k})}^{n}$ is the degree $n$ part of $\Lambda_{N, \mathbb{Q}(\boldsymbol{k})}$, and the inverse system is given by the homomorphism $\Lambda_{M, \mathbb{Q}(\boldsymbol{k})} \rightarrow \Lambda_{N, \mathbb{Q}(\boldsymbol{k})}$ sending $x_{N+1}, \ldots, x_{M}$ to 0 . Under the homomorphism $m_{\lambda}\left(x_{1}, \ldots, x_{M}\right)$ is sent to $m_{\lambda}\left(x_{1}, \ldots, x_{N}\right)$ if $l(\lambda) \leq N$, and to 0 if $l(\lambda)>N$. Therefore Gram-Schmidt orthogonalization is compatible with $N$, and we get $P_{\lambda}^{(\boldsymbol{k})} \in \Lambda_{\mathbb{Q}(\boldsymbol{k})}$, as the limit of $P_{\lambda}^{(\boldsymbol{k})}$ above when $N \rightarrow \infty$. This finishes the proof of the existence of Jack symmetric functions.

For a later purpose, let us give a formula for $D_{N}^{2}$ ([19, VI, §3, Ex.3(d)]). Suppose that $f$ is a homogeneous polynomial of degree $r$. Then

$$
D_{N}^{2} f=\left(-\boldsymbol{k}^{2} U_{N}-\boldsymbol{k} V_{N}+c_{N}\right) f
$$

where

$$
U_{N}=\frac{1}{2} \sum_{i=1}^{N} x_{i}^{2} \frac{\partial^{2}}{\partial x_{i}^{2}}, \quad V_{N}=\sum_{i \neq j} \frac{x_{i}^{2}}{x_{i}-x_{j}} \frac{\partial}{\partial x_{i}}
$$


and

$$
c_{N}=\frac{1}{2} \boldsymbol{k}^{2} r(r-1)+\frac{1}{2} \boldsymbol{k} r N(N-1)+\frac{1}{24} N(N-1)(N-2)(3 N-1) .
$$

We further introduce an operator

$$
\square_{N}^{\boldsymbol{k}} f=\left(\boldsymbol{k} U_{N}+V_{N}-(N-1) r\right) f .
$$

This operator has the limit

$$
\square^{k}=\lim _{N} \square_{N}^{k} .
$$

See [19, VI, §4, Ex.3(a)] or (3.7) below.

We have

$$
\square^{k} P_{\lambda}^{(\boldsymbol{k})}=e_{\lambda}(\boldsymbol{k}) P_{\lambda}^{(\boldsymbol{k})} ; \quad e_{\lambda}(\boldsymbol{k}) \stackrel{\text { def. }}{=} n\left(\lambda^{\prime}\right) \boldsymbol{k}-n(\lambda),
$$

where

$$
n(\lambda)=\sum(i-1) \lambda_{i}=\sum \frac{\lambda_{i}^{\prime}\left(\lambda_{i}^{\prime}-1\right)}{2} .
$$

Computing how $\square^{k}$ acts on the base $\left\{p_{\lambda}\right\}$ of $\Lambda_{\mathbb{Q}(\boldsymbol{k})}$, we obtain the following formula:

$$
\begin{aligned}
\square^{k}=\frac{\boldsymbol{k}}{2} & \sum_{m, n>0} m n p_{m+n} \frac{\partial}{\partial p_{m}} \frac{\partial}{\partial p_{n}}+\frac{\boldsymbol{k}-1}{2} \sum_{m>0} m(m-1) p_{m} \frac{\partial}{\partial p_{m}} \\
& +\frac{1}{2} \sum_{m, n>0}(m+n) p_{m} p_{n} \frac{\partial}{\partial p_{m+n}} .
\end{aligned}
$$

Here we regard $\Lambda_{\mathbb{Q}(\boldsymbol{k})}$ as a polynomial ring $\mathbb{Q}(\boldsymbol{k})\left[p_{1}, p_{2}, \ldots\right]$. This formula will be crucial in $\S 4$. It is not present in [19]. The author learned it from [2] when he wrote [22], but it was certainly known much before.

Remark 3.8. The operator $D_{N}^{2}$ is essentially equal to the CalogeroSutherland hamiltonian, which has been studied intensively in the context of quantum integrable systems. (See [11, §5.5] and the reference therein for example.) It is a trigonometric analog of a quantization of the Calogero-Moser integrable system, appeared in Wilson's work [28], mentioned at [24, a paragraph preceding Theorem 3.46]. At first sight, two appearances of this integrable system have no link: one is a classical system and appears in the deformation of $X^{[n]}$, while the other is quantum and appears in the cohomology of $X^{[n]}$. However, they are connected in a deep way: Bezrukavnikov-Finkelberg-Ginzburg [4] considered the quantized integrable system in positive characteristic, and connected it with the derived category of $X^{[n]}$. See also [3] for a nice application of this result. 
3(iii). Inner product. After giving the review of the definition and basic properties of Jack symmetric functions, we start study of equivariant homology groups of Hilbert schemes. In this subsection we identify (3.1) with the inner product on $\bigoplus H_{T}^{*}\left(X^{[n]}\right)_{\mathbb{F}}$ induced from (2.4).

Let $\alpha, \beta \in H_{T}^{*}(X)_{\mathbb{F}}$. The commutation relation (2.5) implies

$$
\left\langle P_{-n}(\alpha), P_{-n}(\beta)\right\rangle=n\langle\alpha, \beta\rangle .
$$

More generally, we consider an analog of $p_{\lambda}$ :

$$
P_{\lambda}(\alpha)=P_{-\lambda_{1}}(\alpha) P_{-\lambda_{2}}(\alpha) \cdots
$$

for $\lambda=\left(\lambda_{1}, \lambda_{2}, \ldots\right)$. Then we have

$$
\left\langle P_{\lambda}(\alpha), P_{\mu}(\beta)\right\rangle=\delta_{\lambda \mu}\langle\alpha, \beta\rangle^{l(\lambda)} z_{\lambda},
$$

where $z_{\lambda}=\prod k^{m_{k}} m_{k}$ ! for $\lambda=\left(1^{m_{1}} 2^{m_{2}} \cdots\right)$.

In particular, we take $\alpha$ and $\beta$ to be the Poincaré dual of the $x$-axis, i.e., $\alpha=\beta=\varepsilon_{2}$. Then

$$
\langle\alpha, \beta\rangle=-\frac{\varepsilon_{2}}{\varepsilon_{1}}
$$

Substituting this into (3.9) and comparing the result with (3.1), we find

Proposition 3.10. Our inner product is equal to (3.1) used to define Jack symmetric functions under the identification $P_{\lambda}\left(\varepsilon_{2}\right) \leftrightarrow p_{\lambda}$, where the parameter $\boldsymbol{k}$ is $-\varepsilon_{2} / \varepsilon_{1}$.

When $\varepsilon_{1}+\varepsilon_{2}=0$, we have $\boldsymbol{k}=-\varepsilon_{2} / \varepsilon_{1}=1$. It means that our inner product is the standard inner product on symmetric polynomials.

3(iv). Dominance order. Recall that we identify the monomial symmetric function $m_{\lambda}$ with the class $\left[L^{\lambda} C\right]$ in Proposition 2.21. In order to understand the characterization of Jack symmetric functions in (3.3, 3.4), our next task is to explain a geometric meaning of the dominance order (3.2). It is given by modifying the stratification introduced in $[6,13]$.

Let $C=\{\xi=0\}$ as before. For $i \geq 0$, let $\left(\xi^{i}\right)$ be the ideal of functions vanishing to order $\geq i$ along $C$. Let $I \in X^{[n]}$ be an ideal of colength $n$ such that the support of $\mathbb{C}[z, \xi] / I$ is contained in $C$. We consider the sequence $\left(\lambda_{1}^{\prime}, \lambda_{2}^{\prime}, \ldots\right)$ of nonnegative integers given by

$$
\lambda_{i}^{\prime}(I) \stackrel{\text { def. }}{=} \operatorname{dim}\left(\frac{\left(\xi^{i-1}\right)}{I \cap\left(\xi^{i-1}\right)+\left(\xi^{i}\right)}\right) .
$$

The reason why we put the prime become clear later. The sequence in $[6,13]$ was defined by replacing $\left(\xi^{i}\right)$ by $\mathfrak{m}_{x}^{i}$ where $\mathfrak{m}_{x}$ is the maximal 
ideal corresponding to a point $x$. It is clear that $I \supset\left(\xi^{n}\right)$ (see e.g., [13, Lemma 1.1]), hence $\lambda_{i}^{\prime}(I)=0$ for $i \geq n+1$. From the exact sequence

$$
0 \rightarrow \frac{\left(\xi^{i}\right)}{I \cap\left(\xi^{i}\right)} \rightarrow \frac{\left(\xi^{i-1}\right)}{I \cap\left(\xi^{i-1}\right)} \rightarrow \frac{\left(\xi^{i-1}\right)}{I \cap\left(\xi^{i-1}\right)+\left(\xi^{i}\right)} \rightarrow 0,
$$

we have

$$
\sum_{i=1}^{n} \lambda_{i}^{\prime}(I)=n
$$

If $\xi^{i} f_{1}(z), \ldots, \xi^{i} f_{d}(z)$ form a basis of

$$
\frac{\left(\xi^{i}\right)}{I \cap\left(\xi^{i}\right)+\left(\xi^{i+1}\right)},
$$

Then $\xi^{i-1} f_{1}(z), \ldots, \xi^{i-1} f_{d}(z)$ are linearly independent in

$$
\frac{\left(\xi^{i-1}\right)}{I \cap\left(\xi^{i-1}\right)+\left(\xi^{i}\right)} \text {. }
$$

Hence we have $\lambda_{i}^{\prime}(I) \geq \lambda_{i+1}^{\prime}(I)$. Thus $\left(\lambda_{1}^{\prime}(I), \lambda_{2}^{\prime}(I), \ldots\right)$ is a partition of $n$. Let us denote the partition by $\lambda^{\prime}(I)$.

For a partition $\lambda^{\prime}=\left(\lambda_{1}^{\prime}, \lambda_{2}^{\prime}, \ldots\right)$ of $n$, let $V^{\lambda^{\prime}}$ be the set of ideals $I \in X^{[n]}$ such that $\mathbb{C}[z, \xi] / I$ is supported on $C$ and $\lambda^{\prime}(I)=\lambda^{\prime}$. Since

$$
\operatorname{dim} \frac{\left(\xi^{i}\right)}{I \cap\left(\xi^{i}\right)} \leq \sum_{j=i+1}^{n} \lambda_{j}^{\prime}=n-\sum_{j=1}^{i} \lambda_{j}^{\prime}
$$

is a closed condition on $I$, the union

$$
\bigcup_{\mu^{\prime}: \mu^{\prime} \geq \lambda^{\prime}} V^{\mu^{\prime}}
$$

is a closed subset of $\left\{I \in X^{[n]} \mid \operatorname{Supp}(\mathbb{C}[z, \xi] / I) \subset C\right\}$. Thus we have

$$
\text { Closure of } V^{\lambda^{\prime}} \subset \bigcup_{\mu^{\prime} \geq \lambda^{\prime}} V^{\mu^{\prime}}
$$

Suppose that $\lambda^{\prime}$ is the conjugate partition of $\lambda$. We get the following third description of $L^{\lambda} C$.

Proposition 3.13. $L^{\lambda} C=$ Closure of $V^{\lambda^{\prime}}$.

Proof. Let us write $\lambda=\left(\lambda_{1}, \ldots, \lambda_{N}\right)$ with $N=l(\lambda)$. Using the description of $L^{\lambda} C$ as the closure of $W_{\lambda}^{-}$, we first check that $W_{\lambda}^{-} \subset V^{\lambda^{\prime}}$. We may take a generic point in $I \in W_{\lambda}^{-}$, so $\lim _{t \rightarrow \infty} t \cdot I=I_{\left(\lambda_{1}\right), z_{1}} \cap \cdots \cap$ $I_{\left(\lambda_{N}\right), z_{N}}$ such that $x_{i}$ 's are distinct points in $C$ and Young diagrams $D_{1}$, ... have only single column, $D_{1}=\left(\lambda_{1}\right), \ldots$ Since the support of $I$ cannot move as $t \rightarrow \infty$, we can decompose $I=I_{1} \cap \cdots \cap I_{N}$ such that $\operatorname{Supp}\left(\mathbb{C}[z, \xi] / I_{k}\right)=\left\{\left(z_{k}, 0\right)\right\}$. 
Recall that $I_{\left(\lambda_{k}\right), z_{k}}$ is the ideal $\left(z-z_{k}, \xi^{\lambda_{k}}\right)$. It is contained in $V^{\left(\lambda_{k}\right)^{\prime}}$, as can be checked directly in the definition. Since $\left(\lambda_{k}\right)^{\prime}=\left(1^{\lambda_{k}}\right)$ is the unique minimum in the dominance order, $V^{\left(\lambda_{k}\right)^{\prime}}$ is open in $L^{*} C$. As $\lim _{t \rightarrow \infty} t \cdot I_{k}=I_{\left(\lambda_{k}\right), x_{k}}$, we have $t \cdot I_{k} \in V^{\left(\lambda_{k}\right)^{\prime}}$ for sufficiently large $t$. It is clear that $V^{\lambda^{\prime}}$ is invariant under the $\mathbb{C}^{*}$-action for any $\lambda$. In particular, $I_{k} \in V^{\left(\lambda_{k}\right)^{\prime}}$. Since $I=I_{1} \cap \cdots \cap I_{N}$, we have $I \in V^{\lambda^{\prime}}$. Thus $L^{\lambda} C \subset$ Closure of $V^{\lambda^{\prime}}$. As we have $L^{*} C=\bigsqcup V^{\lambda^{\prime}}$, we conclude $L^{\lambda} C=$ Closure of $V^{\lambda^{\prime}}$.

3(v). Fixed points and Jack symmetric functions. Recall that the torus fixed points in $X^{[n]}$ are parametrized by partitions $\lambda$ with $|\lambda|=n$, as we have explained at the beginning of $\S 2(\mathrm{i})$.

Let $\iota:\left(X^{[n]}\right)^{T}=\bigsqcup_{\lambda}\left\{I_{\lambda}\right\} \rightarrow L^{*} C \cap X^{[n]}$ be the inclusion of the $T$-fixed point set. Here note that all fixed points $I_{\lambda}$ are contained in $L^{*} C$. Let $\zeta: L^{*} C \cap X^{[n]} \rightarrow X^{[n]}$ be the inclusion. By the localization theorem, we have isomorphisms

$$
H_{*}^{T}\left(\left(X^{[n]}\right)^{T}\right)_{\mathbb{F}} \stackrel{\iota_{*}}{\cong} H_{*}^{T, l f}\left(L^{*} C \cap X^{[n]}\right)_{\mathbb{F}} \underset{\cong}{\stackrel{\zeta_{*}}{\longrightarrow}} H_{*}^{T, l f}\left(X^{[n]}\right)_{\mathbb{F}} .
$$

The leftmost space $H_{*}^{T}\left(\left(X^{[n]}\right)^{T}\right)_{\mathbb{F}}$ is the direct sum $\bigoplus_{\lambda} \mathbb{Q}\left(\varepsilon_{1}, \varepsilon_{2}\right)\left[I_{\lambda}\right]$, in particular, it has a base $\left[I_{\lambda}\right]$. As $L^{*} C \cap X^{[n]}$ is the union of $W_{\lambda}^{-}$, which is a vector bundle over an affine space $S^{\lambda} C$ by $\S 2($ iii), a standard argument (as in $[24, \mathrm{Ch} .5]$ ) show that $\left[L^{\lambda} C\right]$ is a base of the middle space $H_{*}^{T, l f}\left(L^{*} C \cap X^{[n]}\right)_{\mathbb{F}}$. In particular, we have an isomorphism

$$
\Lambda \otimes \mathbb{Q}\left(\varepsilon_{1}, \varepsilon_{2}\right) \cong H_{*}^{T, l f}\left(L^{*} C\right)_{\mathbb{F}},
$$

extending the isomorphism $\Lambda \cong H_{\text {top }}^{l f}\left(L^{*} C\right)$.

Let $i_{\lambda}:\left\{I_{\lambda}\right\} \rightarrow X^{[n]}$ be the inclusion of the fixed point $I_{\lambda}$ to the Hilbert scheme. Therefore $\zeta \circ \iota=\bigsqcup i_{\lambda}$.

The inverse of the composition $\zeta_{*} \iota_{*}$ in (3.14) is given by

$$
\sum_{\lambda} \frac{1}{e\left(T_{I_{\lambda}} X^{[n]}\right)} i_{\lambda}^{*}(\bullet),
$$

where $e\left(T_{I_{\lambda}} X^{[n]}\right)$ is the $T$-equivariant Euler class of the tangent space of $X^{[n]}$ at $I_{\lambda}$. See Theorem 1.13.

Let us consider the $\iota_{*}^{-1}\left(\left[L^{\lambda} C\right]\right)$. Note that the partition given by (3.11) for $I=I_{\mu}$ is $\mu^{\prime}$. Hence $I_{\mu} \in V^{\mu^{\prime}}$. Therefore we have $I_{\mu} \in L^{\lambda} C$ only if $\lambda \geq \mu$ by (3.12) and Proposition 3.13. This implies that

$$
\iota_{*}^{-1}\left(\left[L^{\lambda} C\right]\right) \in \bigoplus_{\mu \leq \lambda} \mathbb{Q}\left(\varepsilon_{1}, \varepsilon_{2}\right)\left[I_{\mu}\right] .
$$


Let us next consider the coefficient of $\left[I_{\lambda}\right]$ in (3.16). Recall that $L^{\lambda} C$ is defined as the closure of $W_{\lambda}^{-}$in (2.13). As $I_{\lambda} \in S^{\lambda} C, L^{\lambda} C$ is a submanifold in a neighborhood of $I_{\lambda}$. The tangent space $T_{I_{\lambda}} L^{\lambda} C$ is the direct sum of weight subspaces of $T_{I_{\lambda}} X^{[n]}$, whose weights are nonpositive with respect to the $\mathbb{C}^{*}$-action $t(z, \xi)=(z, t \xi)$. Let us decompose the tangent space $T_{I_{\lambda}} X^{[n]}$ into $T_{I_{\lambda}}^{>0} \oplus T_{I_{\lambda}}^{\leq 0}$, sum of positive and nonpositive weight subspaces. Then the fiber of the normal bundle of $L^{\lambda} C$ at $I_{\lambda}$ is identified with $T_{I_{\lambda}}^{>0}$. Hence we have

$$
i_{\lambda *}^{-1} \zeta_{*}\left[L^{\lambda} C\right]=\frac{e\left(T_{I_{\lambda}}^{>0}\right)}{e\left(T_{I_{\lambda}} X^{[n]}\right)}\left[I_{\lambda}\right]=\frac{1}{e\left(T_{I_{\lambda}}^{\leq 0}\right)}\left[I_{\lambda}\right] .
$$

Now we arrive at our main result in this section.

Theorem 3.18. Under the isomorphism (3.14) together with (3.15), the class $\frac{1}{e\left(T_{I_{\lambda}}^{\leq 0}\right)}\left[I_{\lambda}\right]$ corresponds to the Jack symmetric function $P_{\lambda}^{(\boldsymbol{k})}$ with $\boldsymbol{k}=-\varepsilon_{2} / \varepsilon_{1}$.

Proof. Let us check two properties (3.3) and (3.4). The property (3.3) follows from (3.16) and (3.17).

Next note that the composite $\zeta_{*} \iota_{*}$ of (3.14) preserves the inner product by the definition of the inner product (2.4), where the inner product on $H_{*}^{T}\left(\left(X^{[n]}\right)^{T}\right)_{\mathbb{F}} \cong \bigoplus \mathbb{Q}\left(\varepsilon_{1}, \varepsilon_{2}\right)\left[I_{\lambda}\right]$ is the direct sum of the standard inner product $\left\langle\left[I_{\lambda}\right],\left[I_{\lambda}\right]\right\rangle=1$. Then it is clear that $\left\langle\frac{1}{e\left(T_{I_{\lambda}}^{\leq 0}\right)}\left[I_{\lambda}\right], \frac{1}{e\left(T_{I_{\mu}}^{\leq 0}\right)}\left[I_{\mu}\right]\right\rangle=0$ if $\lambda \neq \mu$.

Let us make $e\left(T_{I_{\lambda}}^{\leq 0}\right)$ concrete.

Proposition 3.19. The character of the tangent space of $X^{[n]}$ at the fixed point $I_{\lambda}$ is given by the formula

$$
\operatorname{ch} T_{I_{\lambda}} X^{[n]}=\sum_{s \in \lambda}\left(t_{1}^{l(s)+1} t_{2}^{-a(s)}+t_{1}^{-l(s)} t_{2}^{a(s)+1}\right) .
$$

See [24, Prop. 5.8] for the proof. Here $l(s), a(s)$ are the leg length and the arm length of a square $s$ in the Young diagram corresponding to the partition $\lambda$. Our convention is the same as in [24, (5.7)], and also as in $[19,(6.14)]$ except that our Young diagram is rotated by $90^{\circ}$ in anti-clockwise. For a later purpose, we also introduce the leg colength and the arm colength by $l^{\prime}(s)=i-1, a^{\prime}(s)=j-1$ :

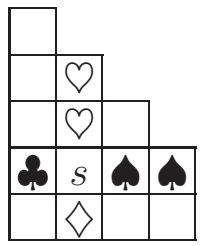

$$
\begin{aligned}
a(s) & =\text { number of } \varnothing \\
l(s) & =\text { number of } \boldsymbol{\phi} \\
a^{\prime}(s) & =\text { number of } \diamond \\
l^{\prime}(s) & =\text { number of } \boldsymbol{\phi}
\end{aligned}
$$


Corollary 3.20. The equivariant Euler class of the nonpositive part $T_{I_{\lambda}}^{\leq 0}$ of the tangent space at the fixed point $I_{\lambda}$ is given by

$$
e\left(T_{I_{\lambda}}^{\leq 0}\right)=\prod_{s \in \lambda}\left((l(s)+1) \varepsilon_{1}-a(s) \varepsilon_{2}\right) .
$$

Comparing this expression with [19, VI, (10.21)], we get

$$
\left[I_{\lambda}\right]=\varepsilon_{1}^{|\lambda|} J_{\lambda}^{(\boldsymbol{k})},
$$

where $J_{\lambda}^{(\boldsymbol{k})}$ is the integral form of the Jack symmetric function $P_{\lambda}^{(\boldsymbol{k})}$, defined in [19, VI, (10.22)].

As a corollary of the above computation, we give a geometric proof of the norm formula [19, VI, (10.16)].

\section{Proposition 3.21.}

$$
\left\langle P_{\lambda}^{(\boldsymbol{k})}, P_{\lambda}^{(\boldsymbol{k})}\right\rangle=\prod_{s \in \lambda} \frac{l(s)+(a(s)+1) \boldsymbol{k}}{l(s)+1+a(s) \boldsymbol{k}} .
$$

Proof. This is a direct consequence of

$$
\left\langle\frac{1}{e\left(T_{I_{\lambda}}^{\leq 0}\right)}\left[I_{\lambda}\right], \frac{1}{e\left(T_{I_{\lambda}}^{\leq 0}\right)}\left[I_{\lambda}\right]\right\rangle=(-1)^{n} \frac{e\left(T_{I_{\lambda}} X^{[n]}\right)}{e\left(T_{I_{\lambda}}^{\leq 0}\right)^{2}}
$$

and the expression of $e\left(T_{I_{\lambda}}^{\leq 0}\right)$ in Corollary 3.20.

3(vi). Nested Hilbert scheme and Pieri formula. Let us first explain the compatibility between the convolution product and the fixed point formula.

Suppose $M_{1}, M_{2}$ are smooth $T$-varieties and $Z \subset M_{1} \times M_{2}$ is a nonsingular $T$-invariant subvariety. We further assume that the second projection $p_{1}: Z \rightarrow M_{1}$ is proper, and hence the convolution product

$$
H_{*}^{T}\left(M_{2}\right) \rightarrow H_{*}^{T}\left(M_{1}\right) ; \bullet \mapsto p_{1 *}\left(p_{2}^{*}(\bullet)\right)
$$

is well-defined. Let $p_{1}^{T}, p_{2}^{T}$ denote the restriction of the first and second projections to the fixed point set $Z^{T}$. respectively.

Let $i_{1}, i_{2}, i_{Z}$ denote the inclusions of fixed point sets $M_{1}^{T}, M_{2}^{T}$, $Z^{T}$ to $M_{1}, M_{2}, Z$ respectively. Let us denote the normal bundles by $N_{1}, N_{2}, N_{Z}$ respectively. We understand that they are union of normal bundles of connected components of $M_{1}^{T}, M_{2}^{T}, Z^{T}$. We do not introduce subscript $\alpha$ unlike in $\S 1($ iv). From Theorem 1.13 we obtain

Lemma 3.22. The following equality holds in $\operatorname{Hom}\left(H_{*}^{T}\left(M_{1}^{T}\right)_{\mathbb{F}}, H_{*}^{T}\left(M_{2}\right)_{\mathbb{F}}\right)$.

$$
p_{1 *} p_{2}^{*} i_{2 *} \frac{1}{e\left(N_{2}\right)}=i_{1 *} p_{1 *}^{T} \frac{1}{e\left(N_{Z}\right)} p_{2}^{T *} .
$$


In fact, we apply Theorem 1.13 to $i_{Z}^{*} p_{2}^{*}=p_{2}^{T *} i_{2}^{*}$ to invert $i_{Z}^{*}, i_{1}^{*}$. Then we use $p_{1 *} i_{Z *}=i_{1 *} p_{1 *}^{T}$. It is suggestive to note that

$$
\frac{e\left(N_{Z}\right)}{p_{2}^{T *} e\left(N_{2}\right)}
$$

is the equivariant Euler class of the virtual normal bundle $N_{Z}-p_{2}^{T *} N_{2}$ of fibers.

We apply this lemma to $P[1] \subset X^{[n]} \times X^{[n-1]} \times X$ in $\S 2(\mathrm{i})$, which realize the operator $P_{-1}(\alpha)$. This $P[1]$ is known to be nonsingular, while other $P[i]$ with $i>1$ are singular except for small $n$. The smoothness was proved in [23] in the context of quiver varieties, and also independently in [7, 26] in the context of Hilbert schemes. It is called the Hecke correspondence [21, 23], and the nested Hilbert scheme in [7] and also various other literature. It has been used to prove many statements on Hilbert schemes by an induction on $n$. See $[10,9]$ for example.

Let us briefly review the proof of smoothness in $[23, \S 5]$. The proof works for higher rank case. We represent $X^{[n]}$ and $X^{[n-1]}$ as spaces of quadruples $\left(B_{1}, B_{2}, i, j\right)$ as in $\left[24\right.$, Ch. 2]: Let $W=\mathbb{C}^{r}, V^{1}=\mathbb{C}^{n}, V^{2}=$ $\mathbb{C}^{n-1}$. Then the framed moduli spaces $M(r, n)(\alpha=1), M(r, n-1)$ $(\alpha=2)$ of torsion free sheaves $(E, \varphi)$ over $\mathbb{P}^{2}$ of rank $r, c_{2}=n, n-1$ are respectively spaces of quadruples $\left(B_{1}^{\alpha}, B_{2}^{\alpha}, i^{\alpha}, j^{\alpha}\right)$ satisfying

- $B_{1}^{\alpha}, B_{2}^{\alpha} \in \operatorname{End}\left(V^{\alpha}\right), i^{\alpha}: W \rightarrow V^{\alpha}, j^{\alpha}: V^{\alpha} \rightarrow W$,

- $\left[B_{1}^{\alpha}, B_{2}^{\alpha}\right]+i^{\alpha} j^{\alpha}=0$,

- (stability) there is no proper subspace of $V^{\alpha}$ containing $i^{\alpha}(W)$ and is invariant under $B_{1}^{\alpha}, B_{2}^{\alpha}$

modulo the conjugation under $\mathrm{GL}\left(V^{\alpha}\right)$. We consider $V^{\alpha}$ as vector bundles over $M(r, n), M(r, n-1)$. We then form a complex of vector bundles over $M(r, n) \times M(r, n-1)$ :

$$
\begin{aligned}
& \operatorname{Hom}\left(V^{1}, Q \otimes V^{2}\right) \\
& \oplus \quad \bigwedge^{2} Q \otimes \operatorname{Hom}\left(V^{1}, V^{2}\right) \\
& \operatorname{Hom}\left(V^{1}, V^{2}\right) \stackrel{a}{\longrightarrow} \quad \begin{array}{r}
\operatorname{Hom}\left(W, V^{2}\right) \\
\oplus
\end{array} \quad \stackrel{b}{\longrightarrow} \quad \begin{array}{c}
\oplus \\
\Lambda^{2} Q \otimes \mathcal{O}
\end{array}, \\
& \operatorname{Hom}\left(V^{1}, \bigwedge^{2} Q \otimes W\right)
\end{aligned}
$$

where $Q=\mathbb{C}^{2}$, and $a, b$ are defined by

$$
\begin{aligned}
& a(\xi \oplus \lambda)=\left(\xi B_{1}^{1}-B_{1}^{2} \xi\right) \oplus\left(\xi B_{2}^{1}-B_{2}^{2} \xi\right) \oplus \xi i^{1} \oplus\left(-j^{2} \xi\right), \\
& b\left(C_{1} \oplus C_{2} \oplus I \oplus J\right)=\left(\begin{array}{c}
B_{1}^{2} C_{2}-C_{2} B_{1}^{1}+C_{1} B_{2}^{1}-B_{2}^{2} C_{1}+i^{2} J+I j^{1} \\
\operatorname{tr}\left(i^{1} J\right)+\operatorname{tr}\left(I j^{2}\right)
\end{array}\right) .
\end{aligned}
$$

This is a complex thanks to $\left[B_{1}^{\alpha}, B_{2}^{\alpha}\right]+i^{\alpha} j^{\alpha}=0$ and $\operatorname{tr}\left(i^{1} j^{2} \xi\right)=$ $\operatorname{tr}\left(\xi i^{1} j^{2}\right)$. 
Lemma 3.24. Consider $a$ and $b$ as linear map between fibers of vector bundles. Then $a$ is injective and $b$ is surjective at any point in $M(r, n) \times$ $M(r, n-1)$.

Proof. See [23, Lemma 5.2]. Note that $V^{1}, V^{2}$ are swapped and the stability condition is the opposite there. Therefore we need to take transposes of $B_{1}^{\alpha}, B_{2}^{\alpha}, i^{\alpha}, j^{\alpha}$.

Therefore Ker $b / \operatorname{Im} a$ forms a vector bundle over $M(r, n) \times M(r, n-1)$ of rank $2 r n-r-1$.

If we omit the factor $\mathcal{O}$ in the third term in (3.23), the complex gives $\operatorname{Ext}^{1}\left(E_{1}, E_{2}\left(-\ell_{\infty}\right)\right)$, where $E_{1}, E_{2}$ are torsion free sheaves in $M(r, n)$ and $M(r, n-1)$ respectively. This is clear from the proof of the description for $M(r, n), M(r, n-1)$. It can be also checked as follows: we consider $M(r, n) \times M(r, n-1)$ as a component of $\mathbb{C}^{*}$-fixed point in $M(2 r, 2 n-1)$, where $\mathbb{C}^{*}$ acts on $\mathbb{C}^{2 r}=\mathbb{C}^{r} \oplus \mathbb{C}^{r}$ with weight 0 on the first factor and 1 on the second. The tangent space of $M(2 r, 2 n-1)$ at $E_{1} \oplus E_{2}$ is $\operatorname{Ext}^{1}\left(E_{1} \oplus E_{2},\left(E_{1} \oplus E_{2}\right)\left(-\ell_{\infty}\right)\right)$, and is a $\mathbb{C}^{*}$-module. The factor $\operatorname{Ext}^{1}\left(E_{1}, E_{2}\left(-\ell_{\infty}\right)\right)$ is the weight 1 subspace. On the other hand, we compute the tangent space in terms of quadruples, and find that (3.23) without $\mathcal{O}$ is the weight 1 subspace.

We define a section $s$ of $\operatorname{Ker} b / \operatorname{Im} a$ by

$$
s=\left(0 \oplus i^{2} \oplus\left(-j^{1}\right)\right) \quad(\bmod \operatorname{Im} a) .
$$

Then $s$ vanishes if and only if there is $\xi$ such that

$$
\xi B_{1}^{1}=B_{1}^{2} \xi, \quad \xi B_{2}^{1}=B_{2}^{2} \xi, \quad \xi i^{1}=i^{2}, \quad j^{1}=j^{2} \xi .
$$

This means we have a homomorphism $E_{1} \rightarrow E_{2}$ which is the identity at $\ell_{\infty}$. It is injective, as a sheaf homomorphism, since it is so at $\ell_{\infty}$. For $r=1$, this means that $I_{1} \subset I_{2}$. The stability condition implies $\xi$ is surjective, hence $\operatorname{Ker} \xi$ is 1-dimensional. The homomorphisms $B_{1}^{1}, B_{2}^{2}$ preserve Ker $\xi$, hence give an element in $\mathbb{C}^{2}=X$. Thus Zero $(s)=P[1]$ if $r=1$, and its natural higher rank generalization for $r>1$.

Now the smoothness of Zero $(s)$ follows from

Lemma 3.25. Take a connection $\nabla$ on $\operatorname{Ker} b / \operatorname{Im} a$ and consider the differential $\nabla s$. It is surjective on $\operatorname{Zero}(s)$.

See [23, Th. 5.7] for the proof. Therefore $\operatorname{Zero}(s)$ is nonsingular of dimension $2 r n-r+1=\frac{1}{2} \operatorname{dim}(M(r, n) \times M(r, n-1) \times X)$.

Now assume $r=1$ and consider a $T^{2}$-action on $P[1]$ induced by $\left(t_{1}, t_{2}\right) \cdot(z, \xi)=\left(t_{1} z, t_{2} \xi\right)$. The fixed points are parametrized by pairs of Young diagrams $(\lambda, \mu)$ of size $n$ and $n-1$ respectively such that $\mu$ 
is contained in $\lambda$, i.e., $\lambda_{i} \geq \mu_{i}$ for all $i \geq 1$. Since the number of boxes differs by 1 , the skew diagram $\lambda-\mu$ consists of a single box.

Using the complex (3.23), we can compute the character of the tangent space at $\left(I_{\lambda}, I_{\mu}\right)$. We consider $V^{1}, V^{2}$ as $T$-modules according to $\lambda, \mu$, and $Q$ as the natural $T$-module. The detail is given in [25, Prop. 5.2]. (Note that $\widehat{M}^{0}(c)$ has no $\mathbb{C}^{2}$-factor, so $t_{1}+t_{2}$ below is not present there.)

\section{Proposition 3.26.}

$$
\operatorname{ch} T_{\left(I_{\mu}, I_{\lambda}\right)} P[1]=t_{1}+t_{2}+\sum_{s \in \mu}\left(t_{1}^{-l_{\lambda}(s)} t_{2}^{a_{\mu}(s)+1}+t_{1}^{l_{\mu}(s)+1} t_{2}^{-a_{\lambda}(s)}\right) .
$$

Here the leg and arm lengths are considered with respect to either $\lambda$ or $\mu$. We use the notation $l_{\lambda}(s), l_{\mu}(s)$ to indicate Young diagrams as subscripts.

Let

$$
b_{\lambda}^{(\boldsymbol{k})}(s) \stackrel{\text { def. }}{=} \frac{\varepsilon_{1}\left(l_{\lambda}(s)+1\right)-\varepsilon_{2} a_{\lambda}(s)}{\varepsilon_{1} l_{\lambda}(s)-\varepsilon_{2}\left(a_{\lambda}(s)+1\right)}=\frac{l_{\lambda}(s)+1+\boldsymbol{k} a_{\lambda}(s)}{l_{\lambda}(s)+\boldsymbol{k}\left(a_{\lambda}(s)+1\right)} .
$$

See $[19,(10.10)]$.

Let us denote $R$ the set of boxes in $\mu$ which lies in the same row with the box $\lambda-\mu$. For example, when $\lambda-\mu$ is the box marked with $\varnothing, R$ consists of boxes with

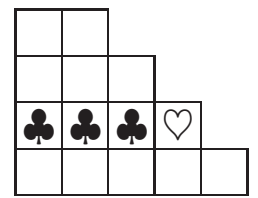

In the notation [19, VI, §6], it is $C_{\lambda / \mu}-R_{\lambda / \mu}$. (Note that our Young diagram is rotated by $90^{\circ}$.)

If $s \in \mu \backslash R$, we have $l_{\lambda}(s)=l_{\mu}(s)$. If $s \in R$, we have $l_{\lambda}(s)=l_{\mu}(s)+1$ and $a_{\lambda}(s)=a_{\mu}(s)$. Therefore

$$
\begin{aligned}
& e\left(T_{\left(I_{\lambda}, I_{\mu}\right)} P[1]\right) \\
= & \varepsilon_{2} e\left(T_{I_{\mu}}^{>0}\right) e\left(T_{I_{\lambda}}^{\leq 0}\right) \prod_{s \in R} \frac{-\varepsilon_{1} l_{\lambda}(s)+\varepsilon_{2}\left(a_{\lambda}(s)+1\right)}{-\varepsilon_{1} l_{\mu}(s)+\varepsilon_{2}\left(a_{\mu}(s)+1\right)} \frac{\varepsilon_{1}\left(l_{\mu}(s)+1\right)-\varepsilon_{2} a_{\mu}(s)}{\varepsilon_{1}\left(l_{\lambda}(s)+1\right)-\varepsilon_{2} a_{\lambda}(s)} \\
= & \varepsilon_{2} e\left(T_{I_{\mu}}^{>0}\right) e\left(T_{I_{\lambda}}^{\leq 0}\right) \prod_{s \in R} \frac{b_{\mu}^{(\boldsymbol{k})}(s)}{b_{\lambda}^{(\boldsymbol{k})}(s)},
\end{aligned}
$$

where $T_{I_{\mu}}^{>0}, T_{I_{\lambda}}^{\leq 0}$ are sum of positive and negative weight spaces as before.

Since $P_{\mu}^{(k)}=\frac{1}{e\left(T_{I_{\mu}}^{\leq 0}\right)}\left[I_{\mu}\right]$ and $p_{1}=P_{-1}\left(\varepsilon_{2}\right)$, we get 
Theorem 3.28. We have

$$
p_{1} P_{\mu}^{(\boldsymbol{k})}=\sum_{\lambda} \prod_{s \in R} \frac{b_{\lambda}^{(\boldsymbol{k})}(s)}{b_{\mu}^{(\boldsymbol{k})}(s)} P_{\lambda}^{(\boldsymbol{k})},
$$

where the summation runs over $\lambda$ with $|\lambda|=|\mu|+1$, containing $\mu$.

This is a special case of Pieri formulas for Jack symmetric functions [19, VI, (6.24)]. When $\boldsymbol{k}=1$, we have $b_{\lambda}^{(\boldsymbol{k})}(s)=1$. Then the above is specialized to a classical Pieri formula for Schur functions.

\section{ViRASORO ALGEBRA}

The goal of this section is to construct a representation of the $\mathrm{Vi}$ rasoro algebra on $\bigoplus H_{*}^{T}\left(X^{[n]}\right)$ for $X=\mathbb{C}^{2}$. Lehn [14] constructed a representation for an arbitrary quasiprojective surface $X$. Our proof is completely different from Lehn's, and based on the geometric construction of Jack symmetric functions in the previous section. The key is that the Hamiltonian $\square^{k}$ gives the Virasoro algebra. On the other hand, Chern classes of the tautological bundle $\mathcal{V}$ on $X^{[n]}$ is diagonalized by the fixed points base $\left\{\left[I_{\lambda}\right]\right\}$. Therefore it is, more or less, obvious that $\square^{k}$ is related to $\mathcal{V}$. As far as the author knows, this observation was not mentioned explicitly in the literature before, but it has been well-known among experts.

4(i). Insertions and coproducts. Note that our Heisenberg generators $P_{i}(\alpha)$ are 'colored' by (co)homology classes $\alpha$ of the base space $X$. When we consider a (normal ordered) product of Heisenberg operators, such as a vertex operator, it is sometime natural to color it a single cohomology class instead of multiples of them. Such a coloring is given naturally by considering a coproduct on $H_{T}^{*}(X)$. This was first noticed by Lehn [14] when he considered Virasoro generators, and subsequently used by other people. Here we use a slightly modified version in [20]. We only consider the case $X=\mathbb{C}^{2}$, but note that the framework makes sense for any $X$ with or without the $T$-action.

Let $\Delta: H_{T}^{*}(X) \rightarrow H_{T}^{*}(X) \otimes H_{T}^{*}(X)$ be the adjoint of the cup product $\cup: H_{T}^{*}(X) \otimes H_{T}^{*}(X) \rightarrow H_{T}^{*}(X)$ with respect to $\langle\rangle,(2.6)$, the negative of the intersection pairing. More concretely, it is $H_{T}^{*}(\mathrm{pt})$-linear, and hence is given by

$$
\Delta(1)=-1 \otimes \mathrm{PD}[0]=-1 \otimes \varepsilon_{1} \varepsilon_{2},
$$

where $\mathrm{PD}[0]$ is the Poincaré dual of the class $[0]$. We also consider its iteration

$$
\Delta^{n}(1)=(-1)^{n} \otimes \underbrace{\varepsilon_{1} \varepsilon_{2} \otimes \cdots \otimes \varepsilon_{1} \varepsilon_{2}}_{n \text { times }} .
$$


Note that we can iterate $\Delta$ in various ways, say $(\Delta \otimes 1) \Delta,(1 \otimes \Delta) \Delta$, but the result is the same thanks to the coassociativity. Thus we have $\Delta^{n}(1)=\left(-\varepsilon_{1} \varepsilon_{2}\right)^{n} \cdot 1 \otimes \cdots \otimes 1$.

We define

$$
\left(P_{m} P_{n}\right)(\alpha) \stackrel{\text { def. }}{=} \sum_{i} P_{m}\left(\alpha_{i}^{\prime}\right) P_{n}\left(\alpha_{i}^{\prime \prime}\right)
$$

if $\Delta(\alpha)=\sum \alpha_{i}^{\prime} \otimes \alpha_{i}^{\prime \prime}$. Similarly we define

$$
: P_{m} P_{n}:(\alpha) \stackrel{\text { def. }}{=} \sum_{i}: P_{m}\left(\alpha_{i}^{\prime}\right) P_{n}\left(\alpha_{i}^{\prime \prime}\right):
$$

Products of more than two operators are defined in the same way, using $\Delta^{n}(\alpha)$.

Remark 4.1. Our $\Delta$ is the negative of $\delta: H_{T}^{*}(X) \rightarrow H_{T}^{*}(X) \otimes H_{T}^{*}(X)$, the pushforward homomorphism associated with the diagonal embedding, used in [14, §3.1].

4(ii). The first Chern class of the tautological bundle. Let $\mathcal{Z}$ be the universal family on $X^{[n]}$, which is a subvariety of $X \times X^{[n]}$. Let $p$ denote the projection to $X^{[n]}$. Then $p_{*} \mathcal{O}_{\mathcal{Z}}$ is a vector bundle of rank $n$ over $X^{[n]}$. In the description [24, Th. 1.9], it is the vector bundle associated with the principal $G L_{n}(\mathbb{C})$-bundle $\widetilde{H} \rightarrow X^{[n]}=\widetilde{H} / G L_{n}(\mathbb{C})$ with respect to the vector representation of $G L_{n}(\mathbb{C})$. Let us denote it by $\mathcal{V}$. It is denoted by $\mathcal{O}^{[n]}$ in [14]. It is called the tautological bundle.

We consider the multiplication of the first Chern class $c_{1}(\mathcal{V})$ of $\mathcal{V}$ as an operator on $H_{T}^{*}\left(X^{[n]}\right)$.

Lemma 4.2. In the fixed points base $\left\{\left[I_{\lambda}\right]\right\}$, the operator $c_{1}(\mathcal{V}) \cup \bullet$ is diagonalized:

$$
c_{1}(\mathcal{V}) \cup\left[I_{\lambda}\right]=-\left(n(\lambda) \varepsilon_{1}+n\left(\lambda^{\prime}\right) \varepsilon_{2}\right)\left[I_{\lambda}\right] .
$$

Proof. Since $\left[I_{\lambda}\right]$ is the class of a fixed point, $c_{1}(\mathcal{V}) \cup\left[I_{\lambda}\right]$ is given by $c_{1}\left(\left.\mathcal{V}\right|_{I_{\lambda}}\right)\left[I_{\lambda}\right]$. The formula of the character of $\left.\mathcal{V}\right|_{I_{\lambda}}$ is given in the proof of [24, Prop. 5.8]. Then the equivariant first Chern class is

$$
c_{1}\left(\left.\mathcal{V}\right|_{I_{\lambda}}\right)=-\sum_{s \in \lambda}\left(l^{\prime}(s) \varepsilon_{1}+a^{\prime}(s) \varepsilon_{2}\right)=-n(\lambda) \varepsilon_{1}-n\left(\lambda^{\prime}\right) \varepsilon_{2} .
$$

The above lemma is easy to prove, but has the following remarkable consequence.

Corollary 4.4. $c_{1}(\mathcal{V}) \cup \bullet$ is equal to $\varepsilon_{1} \square^{\boldsymbol{k}}$ under the isomorphisms $(3.14,3.15)$, where $\square^{k}$ is given by (3.5). 
Proof. Recall that $\square^{k}$ is diagonalized in Jack symmetric functions $P_{\lambda}^{(\boldsymbol{k})}$ with eigenvalues $e_{\lambda}(\boldsymbol{k})=n\left(\lambda^{\prime}\right) \boldsymbol{k}-n(\boldsymbol{k})$, see (3.6). Since $P_{\lambda}^{(\boldsymbol{k})}$ corresponds to the (normalized) fixed point class in Theorem 3.18, the assertion follows as $\varepsilon_{1} e_{\lambda}(\boldsymbol{k})=c_{1}\left(\left.\mathcal{V}\right|_{I_{\lambda}}\right)$ in $(4.3)$.

Let us write $\square^{k}$ in terms of Heisenberg generators $P_{m}(\alpha)$. Recall our identification Proposition 3.10 and the commutation relation $\left[P_{i}\left(\varepsilon_{2}\right), P_{j}\left(\varepsilon_{2}\right)\right]=i \delta_{i+j, 0} \boldsymbol{k}$ id, we rewrite $(3.7)$ by

$$
p_{m} \leftrightarrow P_{-m}\left(\varepsilon_{2}\right), \quad m \frac{\partial}{\partial p_{m}} \leftrightarrow \frac{1}{k} P_{m}\left(\varepsilon_{2}\right)=-P_{m}\left(\varepsilon_{1}\right) .
$$

We get

$$
\begin{aligned}
& \varepsilon_{1} \square^{k} \\
& =-\sum_{m, n>0}\left(\frac{\varepsilon_{2}}{2} P_{-m-n}\left(\varepsilon_{2}\right) P_{m}\left(\varepsilon_{1}\right) P_{n}\left(\varepsilon_{1}\right)+\frac{\varepsilon_{1}}{2} P_{-m}\left(\varepsilon_{2}\right) P_{-n}\left(\varepsilon_{2}\right) P_{m+n}\left(\varepsilon_{1}\right)\right) \\
& \quad+\frac{\varepsilon_{1}+\varepsilon_{2}}{2} \sum_{m>0}(m-1) P_{-m}\left(\varepsilon_{2}\right) P_{m}\left(\varepsilon_{1}\right) .
\end{aligned}
$$

Noticing $\Delta(1)=\varepsilon_{1} \varepsilon_{2}, \Delta^{2}(1)=\left(\varepsilon_{1} \varepsilon_{2}\right)^{2}$, and $K_{X}=-\varepsilon_{1}-\varepsilon_{2}$, we rewrite this expression as follows. $\left(P_{0}(\alpha)\right.$ is understood as 0 .) It is a special case of Lehn's result which holds for any $X$.

Theorem 4.5 (Lehn [14]).

$$
\begin{aligned}
c_{1}(\mathcal{V}) \cup \bullet=-\frac{1}{3 !} \sum_{m_{1}+m_{2}+m_{3}=0}: & P_{m_{1}} P_{m_{2}} P_{m_{3}}:(1) \\
& +\frac{1}{4} \sum_{m}(|m|-1): P_{-m} P_{m}:\left(K_{X}\right) .
\end{aligned}
$$

The commutator with $P_{n}(\alpha)$ is given by

$$
\left[c_{1}(\mathcal{V}) \cup \bullet, P_{n}(\alpha)\right]=\frac{n}{2} \sum_{l+m=n}: P_{l} P_{m}:(\alpha)-\frac{n(|n|-1)}{2} P_{n}\left(K_{X} \cup \alpha\right) .
$$

The formula is presented in this form in [14, Th. 3.10] up to the sign convention. Since $\bigoplus_{n} H_{*}^{T}\left(X^{[n]}\right)$ is an irreducible representation of the Heisenberg algebra, two formulas are equivalent.

Remark 4.6. Note that $\mathfrak{q}_{n}(\alpha)$ in [14] is our $P_{-n}(\alpha)$.

The terms

$$
L_{n}(\alpha) \stackrel{\text { def. }}{=} \frac{1}{2} \sum_{l+m=n}: P_{l} P_{m}:(\alpha)
$$


satisfy the Virasoro relation

$$
\left[L_{n}(\alpha), L_{m}(\beta)\right]=(n-m) L_{n+m}(\alpha \beta)-\frac{n^{3}-n}{12} \delta_{n+m, 0}\left\langle c_{2}(X) \alpha, \beta\right\rangle \mathrm{id},
$$

where $c_{2}(X)=\varepsilon_{1} \varepsilon_{2}$. It appears as the composite

$$
H_{T}^{*}(X) \stackrel{\Delta}{\rightarrow} H_{T}^{*}(X) \otimes H_{T}^{*}(X) \stackrel{\cup}{\rightarrow} H_{T}^{*}(X)
$$

is multiplication with $-c_{2}(X)$. We leave a check of $(4.7)$ to the reader.

Remark 4.8. It would be nice if one could deduce Lehn's result for general $X$ from Theorem 4.5. In fact, Li-Qin-Wang [16] (see also [15]) proved that operators given by cup products of classes

$$
p_{1 *}\left(\operatorname{ch}\left(\mathcal{O}_{\mathcal{Z}_{n}}\right) p_{2}^{*}\left(\operatorname{td}_{X} \cup \alpha\right)\right),
$$

are expressed by universal polynomials in Heisenberg operators coupled with $\alpha \cup\left\{1_{X}, K_{X}, K_{X}^{2}, c_{2}(X)\right\}$, independent of $X$. Here $\mathcal{Z}_{n}$ is the universal subscheme in $X^{[n]} \times X, p_{1}, p_{2}$ are projection from $X^{[n]} \times X$ to $X^{[n]}$ and $X$ respectively, and $\operatorname{td}_{X}$ is the Todd class of $X$. By RiemannRoch, $c_{1}(\mathcal{V})$ is the degree 2 part of the above for $\alpha=1_{X}$. Thanks to the universality, it is enough to determine the polynomials for $X=\mathbb{C}^{2}$. However the universality in [16] was derived from Lehn's result, and hence this argument does not work.

\section{REFERENCES}

[1] M. Audin, Torus actions on symplectic manifolds, revised ed., Progress in Mathematics, vol. 93, Birkhäuser Verlag, Basel, 2004.

[2] H. Awata, Y. Matsuo, S. Odake, and J. Shiraishi, Collective field theory, Calogero-Sutherland model and generalized matrix models, Phys. Lett. B 347 (1995), no. 1-2, 49-55.

[3] R. Bezrukavnikov and M. Finkelberg, Wreath Macdonald polynomials and categorical McKay correspondence, August 2012, with an appendix by Vadim Vologodsky, arXiv:1208.3696 [math.RT].

[4] R. Bezrukavnikov, M. Finkelberg, and V. Ginzburg, Cherednik algebras and Hilbert schemes in characteristic p, Represent. Theory 10 (2006), 254-298, With an appendix by Pavel Etingof.

[5] A. Białynicki-Birula, Some theorems on actions of algebraic groups, Ann. of Math. (2) 98 (1973), 480-497.

[6] J. Briançon, Description de $\operatorname{Hilb}^{n} C\{x, y\}$, Invent. Math. 41 (1977), no. 1, 45-89.

[7] J. Cheah, Cellular decompositions for nested Hilbert schemes of points, Pacific J. Math. 183 (1998), no. 1, 39-90.

[8] D. Edidin and W. Graham, Equivariant intersection theory, Invent. Math. 131 (1998), no. 3, 595-634.

[9] G. Ellingsrud, L. Göttsche, and M. Lehn, On the cobordism class of the Hilbert scheme of a surface, J. Algebraic Geom. 10 (2001), no. 1, 81-100. 
[10] G. Ellingsrud and S. A. Strømme, An intersection number for the punctual Hilbert scheme of a surface, Trans. Amer. Math. Soc. 350 (1998), no. 6, 25472552.

[11] P. Etingof, Calogero-Moser systems and representation theory, Zurich Lectures in Advanced Mathematics, European Mathematical Society (EMS), Zürich, 2007.

[12] M. Haiman, Hilbert schemes, polygraphs and the Macdonald positivity conjecture, J. Amer. Math. Soc. 14 (2001), no. 4, 941-1006 (electronic).

[13] A. A. Iarrobino, Punctual Hilbert schemes, Mem. Amer. Math. Soc. 10 (1977), no. 188 , viii +112 .

[14] M. Lehn, Chern classes of tautological sheaves on Hilbert schemes of points on surfaces, Invent. Math. 136 (1999), no. 1, 157-207.

[15] - Lectures on Hilbert schemes, Algebraic structures and moduli spaces, CRM Proc. Lecture Notes, vol. 38, Amer. Math. Soc., Providence, RI, 2004, pp. 1-30.

[16] W.-P. Li, Z. Qin, and W. Wang, Stability of the cohomology rings of Hilbert schemes of points on surfaces, J. Reine Angew. Math. 554 (2003), 217-234.

[17] _ The cohomology rings of Hilbert schemes via Jack polynomials, Algebraic structures and moduli spaces, CRM Proc. Lecture Notes, vol. 38, Amer. Math. Soc., Providence, RI, 2004, pp. 249-258.

[18] G. Lusztig, Cuspidal local systems and graded Hecke algebras. I, Inst. Hautes Études Sci. Publ. Math. (1988), no. 67, 145-202.

[19] I. G. Macdonald, Symmetric functions and Hall polynomials, second ed., Oxford Mathematical Monographs, The Clarendon Press Oxford University Press, New York, 1995, With contributions by A. Zelevinsky, Oxford Science Publications.

[20] D. Maulik and A. Okounkov, Quantum groups and quantum cohomology, arXiv:1211.1287.

[21] H. Nakajima, Instantons on ALE spaces, quiver varieties, and Kac-Moody algebras, Duke Math. J. 76 (1994), no. 2, 365-416.

[22] _ Jack polynomials and Hilbert schemes of points on surfaces, October 1996, arXiv: alg-geom/9610021.

[23] _ Quiver varieties and Kac-Moody algebras, Duke Math. J. 91 (1998), no. $3,515-560$.

[24] Lectures on Hilbert schemes of points on surfaces, University Lecture Series, vol. 18, American Mathematical Society, Providence, RI, 1999.

[25] H. Nakajima and K. Yoshioka, Perverse coherent sheaves on blow-up. II. Wallcrossing and Betti numbers formula, J. Algebraic Geom. 20 (2011), no. 1, $47-100$.

[26] A. S. Tikhomirov, The variety of complete pairs of zero-dimensional subschemes of an algebraic surface, Izv. Ross. Akad. Nauk Ser. Mat. 61 (1997), no. $6,153-180$.

[27] E. Vasserot, Sur l'anneau de cohomologie du schéma de Hilbert de $\mathbf{C}^{2}$, C. R. Acad. Sci. Paris Sér. I Math. 332 (2001), no. 1, 7-12.

[28] G. Wilson, Collisions of Calogero-Moser particles and an adelic Grassmannian, Invent. Math. 133 (1998), no. 1, 1-41, With an appendix by I. G. Macdonald. 
Research Institute for Mathematical Sciences, Kyoto University, КYОTO 606-8502, JAPAN

E-mail address: nakajima@kurims.kyoto-u.ac.jp 